\title{
Serum- and glucocorticoid- inducible kinase 2 , SGK2, is a novel autophagy regulator and modulates platinum drugs response in cancer cells
}

\author{
Valentina Ranzuglia ${ }^{1}$ - Ilaria Lorenzon ${ }^{1} \cdot$ Ilenia Pellarin ${ }^{1} \cdot$ Maura Sonego $^{1} \cdot$ Alessandra Dall'Acqua $^{1} \cdot$ Sara D'Andrea $^{1}$ \\ Sara Lovisa ${ }^{1} \cdot$ Ilenia Segatto ${ }^{1}$. Michela Coan ${ }^{1} \cdot$ Jerry Polesel $^{2}$ - Diego Serraino ${ }^{2}$ - Patrizia Sabatelli, ${ }^{3,4}$. \\ Paola Spessotto $\mathbb{D}^{1} \cdot$ Barbara Belletti $\mathbb{D}^{1} \cdot$ Gustavo Baldassarre $\mathbb{D}^{1} \cdot$ Monica Schiappacassi $\mathbb{D}^{1}$
}

Received: 30 December 2019 / Revised: 7 August 2020 / Accepted: 13 August 2020 / Published online: 27 August 2020

(c) The Author(s) 2020. This article is published with open access

\begin{abstract}
For many tumor types chemotherapy still represents the therapy of choice and many standard treatments are based on the use of platinum (PT) drugs. However, de novo or acquired resistance to platinum is frequent and leads to disease progression. In Epithelial Ovarian Cancer (EOC) patients, PT-resistant recurrences are very common and improving the response to treatment still represents an unmet clinical need. To identify new modulators of PT-sensitivity, we performed a loss-of-function screening targeting 680 genes potentially involved in the response of EOC cells to platinum. We found that SGK2 (Serum-and Glucocorticoid-inducible kinase 2) plays a key role in PT-response. We show here that EOC cells relay on the induction of autophagy to escape PT-induced death and that SGK2 inhibition increases PT sensitivity inducing a block in the autophagy cascade due to the impairment of lysosomal acidification. Mechanistically we demonstrate that SGK2 controls autophagy in a kinase-dependent manner by binding and inhibiting the V-ATPase proton pump. Accordingly, SGK2 phosphorylates the subunit V1H (ATP6V1H) of V-ATPase and silencing or chemical inhibition of SGK2, affects the normal autophagic flux and sensitizes EOC cells to platinum. Hence, we identified a new pathway that links autophagy to the survival of cancer cells under platinum treatment in which the druggable kinase SGK2 plays a central role. Our data suggest that blocking autophagy via SGK2 inhibition could represent a novel therapeutic strategy to improve patients' response to platinum.
\end{abstract}

These authors contributed equally: Valentina Ranzuglia, Ilaria Lorenzon, and Ilenia Pellarin

These authors jointly supervised this work: Gustavo Baldassarre and Monica Schiappacassi

Supplementary information The online version of this article (https:// doi.org/10.1038/s41388-020-01433-6) contains supplementary material, which is available to authorized users.

Gustavo Baldassarre
gbaldassarre@cro.it
Monica Schiappacassi
mschiappacassi@cro.it
Division of Molecular Oncology, Centro di Riferimento
Oncologico di Aviano (CRO), IRCCS, 33081 Aviano, Italy

\section{Introduction}

Platinum-based chemotherapy is employed in the treatment of a wide range of solid tumors, including head and neck, pancreas, colorectal, bladder, ovarian, and lung cancer $[1,2]$. Despite a good rate of initial responses, platinumbased treatment often results in the development of chemoresistant recurrences leading to treatment failure [3-8]. This issue is particularly relevant for Epithelial Ovarian Cancer (EOC) where acquired resistance to platinum (PT) is a frequent event that predicts poor prognosis for patients $[9,10]$. During the last years, several comprehensive

2 Epidemiology, Centro di Riferimento Oncologico di Aviano (CRO), IRCCS, 33081 Aviano, Italy

3 Institute of Molecular Genetics, National Research Council, Bologna, Italy

4 Rizzoli Orthopedic Institute, Bologna, Italy 
genomic analyses of EOC have been performed to reveal new oncogenic drivers and possible mediators of PTresistance in the most common histotypes [11-13]. These studies underscored the heterogeneity of EOC and the need of novel approaches to identify new possible therapeutic targets.

Many studies have been done to identify genes and mechanisms directly associated to resistance to PT therapy. PT-resistance has been linked to alterations in several processes such as drug transport, drug inactivation, DNA damage response, DNA repair, and apoptosis [14, 15]. Among general stress response pathways autophagy has also been associated to PT-resistance [16, 17]. In fact, autophagy has been shown to confer cancer cells the metabolic plasticity necessary to grow in suboptimal environments and to survive under therapy-induced stress [18-21].

We performed a loss-of function screening to identify genes able to impact on PT treatment targeting the pathways linked to PT-resistance in EOC. Using this approach, we unveiled SGK2, serum-and glucocorticoid- kinase 2, as a novel modulator of platinum sensitivity.

The SGK family is composed of three isoforms: SGK1, SGK2, and SGK3, belonging to the AGC kinase group $[22,23]$. AGC family comprises 60 members, some of them extensively studied in cancer research such as AKT, S6K, and RSK. Literature data indicate that the three SGK isoforms share similar biochemical properties and structure, with $80 \%$ homology in their kinase domains, $44-68 \%$ in their carboxy termini non-catalytic domain and $25 \%$ at the NH2-terminus between SGK1 and SGK3 and almost no identity between SGK2 and the other isoforms [24]. Most of the scientific knowledge about SGK family roles in cellular physiology and in the development of human diseases is based on the study of SGK1 and SGK3 genes, while SGK2 is a virtually uncharacterized one. SGKs were initially identified as regulators of several transporters, channels, and pumps in the context of epithelial ions transport [25-28]. Recent works indicate that SGK1 and SGK3 have an emerging role in cancer biology sustaining tumor growth in presence of $\mathrm{PI} 3 \mathrm{~K} / \mathrm{AKT}$ inhibition and suggest that these genes could have an oncogenic function [29-32].

In the current study, we have demonstrated a previously unrecognized role of SGK2 in PT-sensitivity exerted by the modulation of autophagic flux.

\section{Results}

\section{SGK2 silencing sensitizes EOC cells to platinum}

To unveil key genes involved in the regulation of PT sensitivity of EOC cells we used a functional genomic approach targeting 680 genes related to apoptosis, p53 and
DNA repair pathways with three shRNAs in two different EOC cell lines (MDAH-2774, herein MDAH, and SKOV3) (Fig. 1a and S1a). Statistical analysis and quality controls of this high-throughput screening identified 50 genes as possible PT-sensitizers (screening raw and analyzed data is reported in Data File S1 and Supplementary Materials and Methods). A subsequent screening using four different EOC cell lines was performed to validate these candidate genes (Data File S1, Section 8). Among the confirmed ones $[33,34]$ (described in Section 9, Data File S1), SGK2 silencing showed increased PT-induced death in three out of four different EOC cell lines used (i.e., MDAH, OV90 and SKOV3 but not in TOV112D) (Fig. S1b).

SGK2 expression (mRNA and protein) was analyzed in normal human epithelial ovarian cells (HuNoEOC) and in a panel of seven available EOC cell lines showing a good concordance between RNA and protein expression (Fig. 1b, c). Interestingly, SGK2 was not expressed in the TOV112D cell line (used in the validation screening) giving a possible explanation for the fact that SGK2 shRNAs did not affect PT-induced death in this cell line (Fig. S1b), although no correlation was observed between SGK2 expression and the PT-IC50 of the analyzed cells. To confirm the screening results, we used SGK2 expressing cells (MDAH, TOV21G, SKOV3, and OV90) and treated them with carboplatin (CBDCA). In all models SGK2 silencing significantly increased CBDCA-induced death, although some variability among the different shRNA was observed (Fig. 1d and S1c-e). Accordingly, overexpression of SGK2 in OVCAR8 cells (where endogenous SGK2 expression was barely detectable) increased the resistance to CBDCA (Fig. 1e). Moreover, SGK2 silencing in MDAH and TOV21G cells significantly decreased their CBDCA IC50 as evaluated by dose-response curves (Fig. S2a).

Given the high sequence homology described for the three members of the SGK family $[24,35]$ we also analyzed the expression of SGK1 and SGK3 in normal and cancer-derived EOC cells and tested if their silencing could have any role in PT-sensitivity. SGK3 was highly expressed in almost all tested cells while SGK1 was expressed in 6/7 tested EOC cell lines. Both proteins were easily detected in normal HuNoEOC (Fig. 1c). Using MDAH as a model, we observed that silencing of SGK3 and SGK1 was specific and did not affect their survival and the PT-sensitivity (Fig. S2b, c).

\section{SGK2 silencing sensitizes EOC cells to platinum via a kinase-dependent mechanism}

To understand how SGK2 regulates the PT-sensitivity in EOC cells, we first investigated if its kinase activity was involved. To this aim, we generated a SGK2 kinase-dead (KD) mutant by substituting the Lys64 residue within the ATP binding region with a methionine, as described by 
Fig. 1 SGK2 silencing sensitizes ovarian cancer cells to platinum treatment. a Experimental design of the loss-of-function screening. Transduced cells were treated or not with CBDCA for $16 \mathrm{~h}$ using a dose able to induce only $10-20 \%$ of cell mortality. b SGK2 mRNA expression in the indicated EOC cell lines evaluated by qRT-PCR. c Western blot (WB) analyses evaluating SGK1, SGK2, and SGK3 expression in the indicated EOC cell lines. Vinculin was used as loading control. d Graph reports the viability of MDAH cells transduced with control (sh Ctrl) and three different

SGK2 shRNAs, and then treated with CBDCA $140 \mu \mathrm{g} / \mathrm{ml}$ for $16 \mathrm{~h}$ as in a. On the right, WB analysis of SGK2, SGK1 and SGK3 expression in SGK2 silenced MDAH cells. e Graph reports the viability of OVCAR8 cells stably overexpressing EGFP-SGK2. Cells were treated with increasing doses of CBDCA and cell viability analyzed as in d. Results are expressed as percentage of CBDCA survived cells between treated and untreated cells (set as $100 \%$ as reference). On the right, WB analyses of SGK2 expression in the used cells. Vinculin was used as loading control. In $\mathbf{d}$ and e data represent the mean $\pm \mathrm{SD}$ of three independent experiments. Significance was calculated using two-tailed, unpaired Student's $t$ test. $* * * p<$ $0.001, * * p<0.01, * p<0.05$. (See also Figs. S1 and S2). a

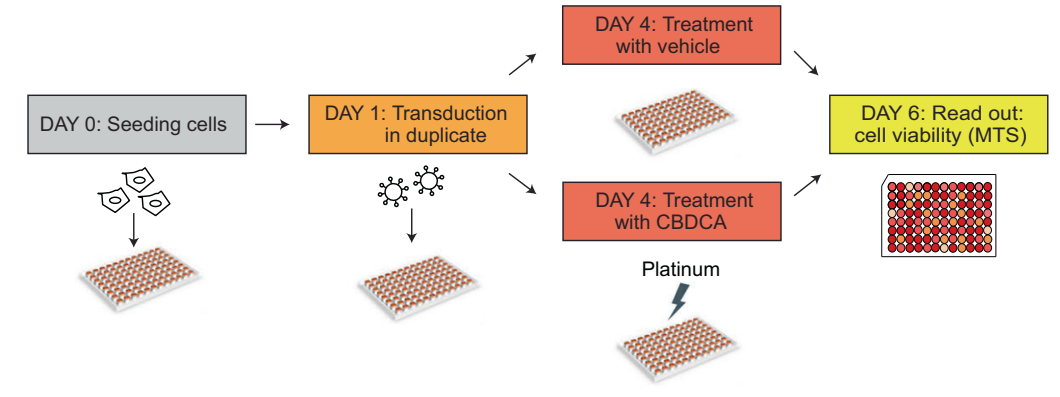

b

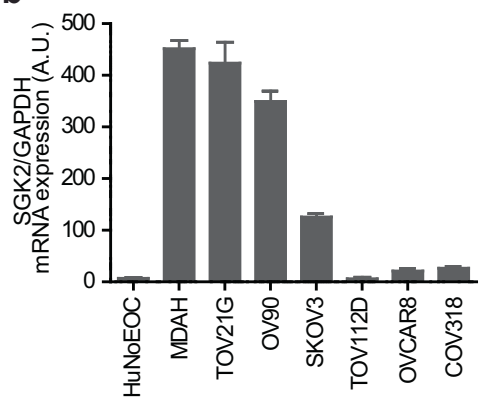

C

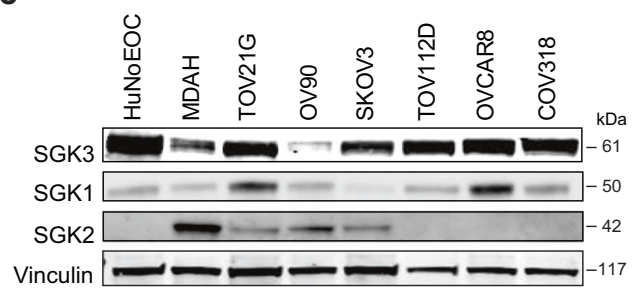

d
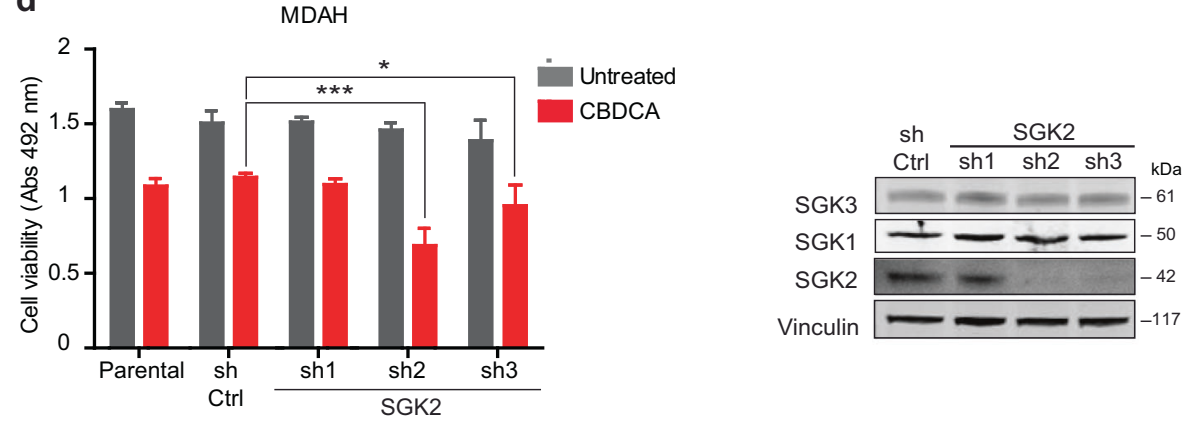

e

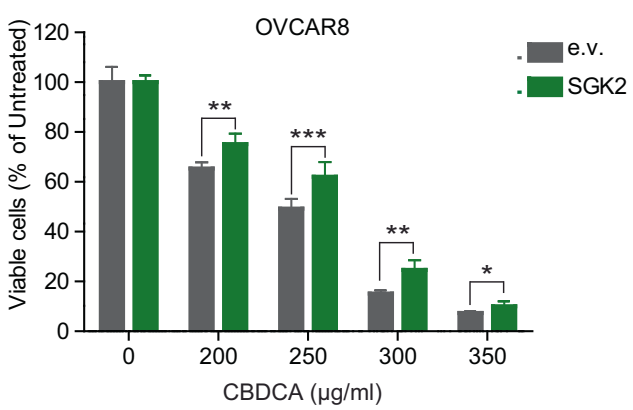

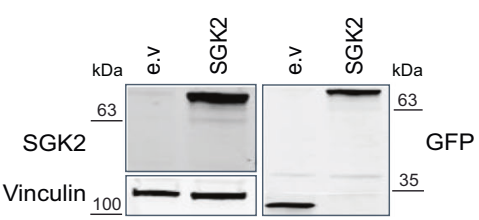

others [28]. This mutant had no effect on PT-sensitivity in TOV21G cells (Fig. S3a), suggesting a possible role for SGK2 kinase activity on PT-induced death. Based on the work of Hemmings and collaborators demonstrating that mutation of Thr256 and Ser422 in alanine confers to SGK1 a dominant-negative (DN) activity [36], we next generated a SGK2 DN construct by substituting with an alanine the corresponding Thr193 and Ser356 residues in the catalytic and activating domain (Fig. 2a). We also substituted Ser 356 in the activating domain with the phosphomimetic residue Asp (S356D) to generate a SGK2 constitutively active (CA) mutant. In dose-response experiments, the overexpression of SGK2 DN increased and SGK2 CA decreased the sensitivity of TOV21G cells to CBDCA (Fig. 2b). Taken together these data support the possibility that SGK2 kinase activity plays a role in the control of PTinduced death. Accordingly, pretreatment of EOC cells for $24 \mathrm{~h}$ with increasing doses (from 35 to $65 \mu \mathrm{M}$ ) of the smallmolecule GSK650394, a specific SGK1/SGK2 inhibitor [37] significantly increased MDAH cells sensitivity to PT 
a

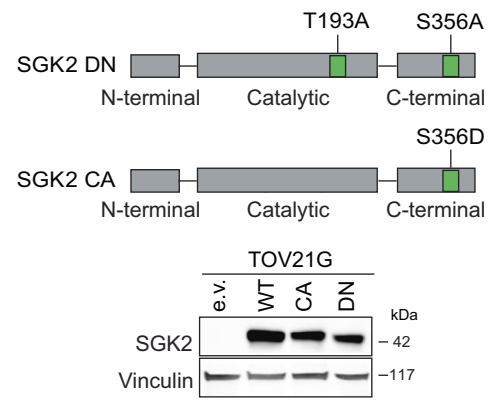

C
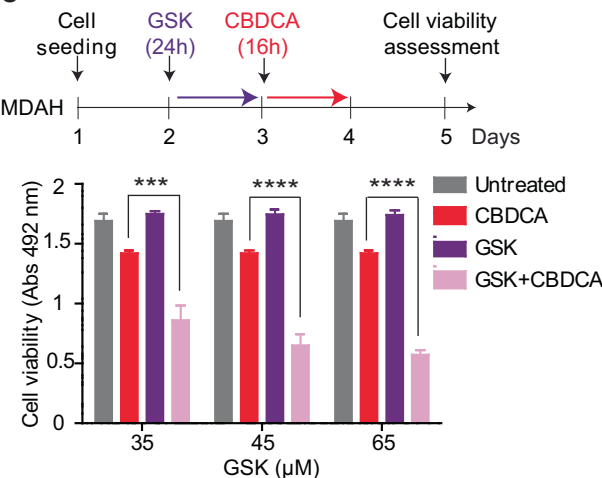

b

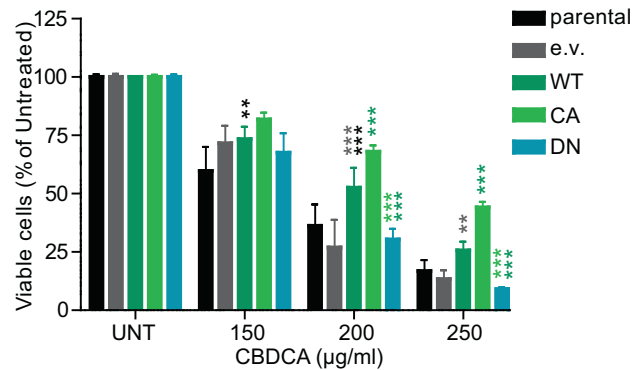

d
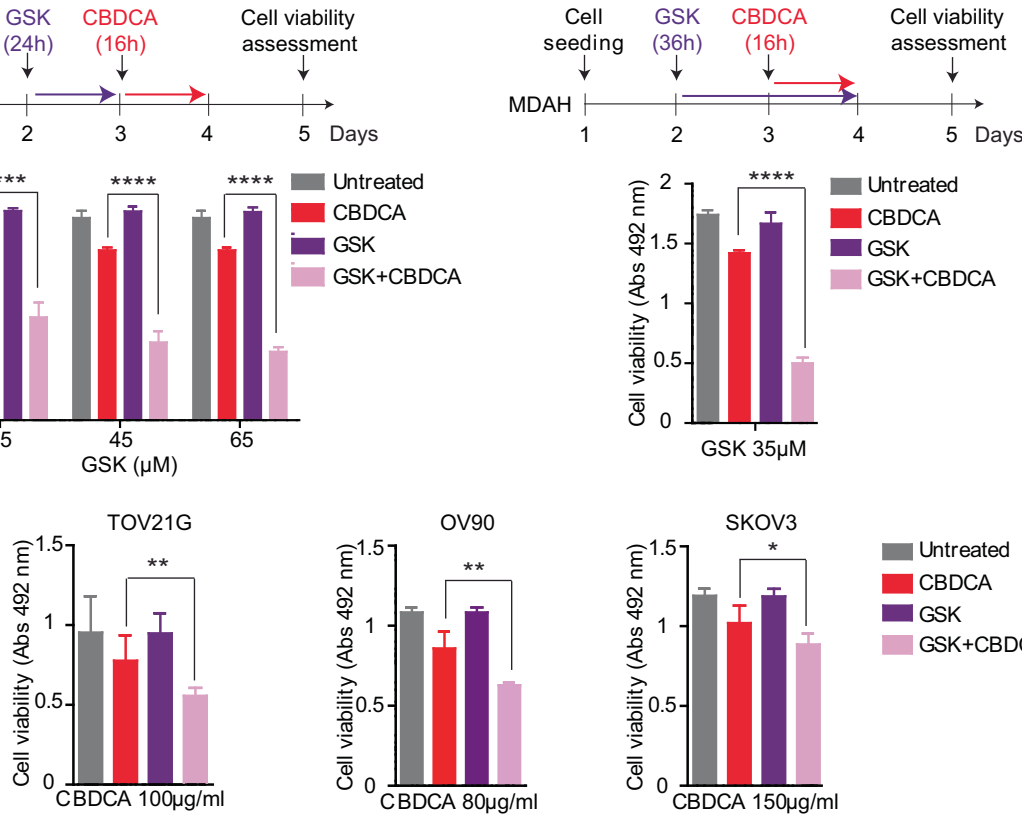

Untreated

CBDCA

GSK

GSK+CBDCA
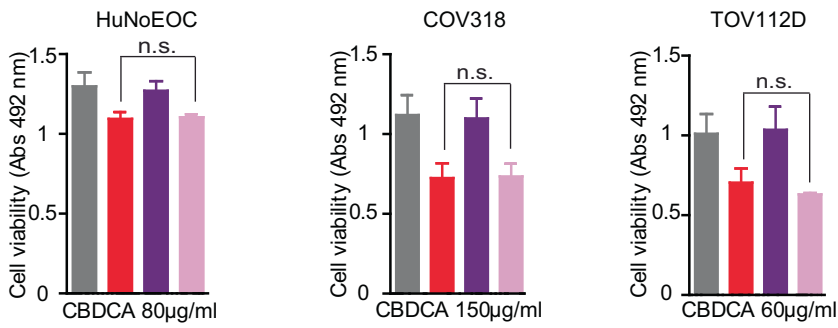

Fig. 2 SGK2 silencing sensitizes ovarian cancer cells to platinum via a kinase-dependent mechanism. a Schematic representation of SGK2 dominant negative (DN) (pLPC-SGK2 ${ }^{\mathrm{T} 193 \mathrm{~A} / \mathrm{S} 356 \mathrm{~A}}$ ) and constitutively active (CA) (pLPC-SGK2 ${ }^{\mathrm{S} 356 \mathrm{D}}$ ) constructs (upper panel). Overexpression of the different SGK2 constructs in TOV21G cells was confirmed by WB (lower panel). Vinculin was used as loading control. b Graph reporting cell viability of TOV $21 \mathrm{G}$ cells described in a and treated with increasing doses of CBDCA. Results are expressed as survival ratio (\%) of CBDCA treated over untreated cells (set as $100 \%$ as reference) (e.v =empty vector). $\mathbf{c}$ Graph reporting cell viability of MDAH cells treated with the indicated doses of GSK650394 (GSK), followed by CBDCA $140 \mu \mathrm{g} / \mathrm{ml}$ as depicted in the experimental

showing no toxic effects when used alone (Fig. 2c). This effect was amplified when GSK650394 was maintained in culture with CBDCA for a total time of $36 \mathrm{~h}$, again with no timeline shown above the graph. d Graph reporting cell viability of MDAH cells treated for $36 \mathrm{~h}$ with GSK $35 \mu \mathrm{M}$, in the presence of CBDCA $140 \mu \mathrm{g} / \mathrm{ml}$ for the last $16 \mathrm{~h}$ as depicted in the shown experimental timeline. e Graphs reporting cell viability of SGK2-expressing (TOV21G, OV90, and SKOV3) and SGK2 not-expressing cell lines (HuNoEOC, COV318, TOV112D) treated with GSK $35 \mu \mathrm{M}$, CBDCA at the indicated doses used alone or in combination using the timeline reported in d. Data represent the mean $\pm \mathrm{SD}$ of three independent experiments. Significance was calculated using two-tailed, unpaired Student's $t$ test. $* * * * p<0.0001, * * * p<0.001, * * p<0.01$, ns not significant. (See also Fig. S3).

appreciable toxicity (Fig. 2d). A similar increase in PTsensitivity was observed using cisplatin (CDDP) in place of CBDCA (Fig. S3b). Accordingly, GSK650394 significantly 
decreased CBDCA IC50 in dose-response analyses performed in MDAH and TOV $21 \mathrm{G}$ but not in COV318 cells that do not express SGK2 (Fig. S3c, d). Using this approach, we identified the $35 \mu \mathrm{M}$ dose as the most effective to be used in combination with CBDCA in all tested cells (Fig. S3c). Next, by using the combined GSK650394 + CBDCA treatment on a panel of EOC cells we verified that GSK650394 increased PT-sensitivity in SGK2 expressing cells (MDAH, TOV21G, OV90, and SKOV3) but not in those that did not express appreciable levels of SGK2 protein (COV318, TOV112D, and normal HuNoEOC) (Fig. 2e). Similarly, GSK650394 did not have any effect on PT-sensitivity in TOV21G cells stably expressing SGK2 DN (Fig. S3e). These results indicated that the GSK650394 inhibitor sensitized EOC cells to CBDCA in a SGK2-dependent manner and confirmed the role of SGK2 kinase activity in the control of PT-sensitivity.

\section{SGK2 inhibition also improves platinum response in platinum-resistant EOC cells, primary EOC cells and breast and head and neck cancer cells}

We next explored if SGK2 inhibition could re-sensitize platinum-resistant cells (PT-res) to platinum. To this aim we exploited the isogenic MDAH PT-res cells recently generated in our lab (herein MDAH PT-res) [38]. MDAH PT-res cells had higher levels of SGK2 and the combined GSK650394 + CBDCA treatment induced an increase in MDAH PT-res cell death when compared to CBDCA single treatment at the tested doses (Fig. 3a).

Then we analyzed SGK2 expression and PT-sensitivity in primary cells isolated in our lab from EOC patients' surgical samples. We analyzed four different primary cell lines (Fig. S4) comparing SGK2 mRNA expression to EOC cell lines, setting as cut off value the expression of SGK2 in the SKOV3 cells (which had the lowest SGK2 expression detectable by western blot, Figs. 1c and 3b). The 49d and 66 primary EOC cells that displayed the highest SGK2 expression also had the highest CDDP IC50 (Fig. 3c), supporting a possible correlation between SGK2 expression and response to PT in primary cultures.

Moreover, the GSK650394+CBDCA treatment increased cell death also in Triple-Negative Breast Cancer (TNBC) (MDA-MB-468 and BT-549) and in Head and Neck Squamous Cancer (HNSCC) (FaDu and CAL27) cells (Fig. 3d), two human cancer types known to be treatable with PT in the clinical practice.

\section{SGK2 modulates autophagic flux}

In performing the experiments described above, we observed that GSK650394 induced the formation of cytoplasmic vesicles in EOC cells both when used as single treatment (Fig. 4a, b) and in combination with CBDCA (Fig. S5a, b). This vesicles' formation was noticed only in the SGK2-expressing cell lines (MDAH and TOV21G) but not in TOV112D cells that did not express SGK2 (Fig. $\mathrm{S} 5 \mathrm{~b})$. Vesicles' formation was reversible since the withdrawal of GSK650394 from the medium resulted in their disappearance within $24 \mathrm{~h}$ (Fig. S5c). The direct involvement of SGK2 in the formation of these cytoplasmic vesicles was confirmed by silencing SGK2 in MDAH and TOV21G cells (Fig. S5d), although less pronounced than the one observed with GSK650394. Similar results were recapitulated in all tested TNBC and HNSCC cell lines (Fig. S5e, f).

Transmission Electron Microscopy (TEM) in MDAH and TOV21G cells was next used for a deeper understanding of the nature of these cytoplasmic vesicles. TEM showed that vesicles induced by GSK650394 treatment had an ultrastructure compatible with that of autophagic vacuoles (AV) [39, 40] with typical features of initial autophagic vacuoles (AVi), that could be mostly categorized as autophagosomes (AP) as indicated by the presence of double membrane (Figs. 4a, c and S6a). APs were present in approximately 10\% of GSK650394-treated cells. Moreover, almost all treated cells also presented degradative autophagic vacuoles (AVd) accumulating partially degraded material (Fig. 4c). Neither AVi nor AVd were observed in untreated cells. These data suggested that GSK650394 treatment resulted in the alteration of the autophagic flux likely by affecting the rate at which the autophagy machinery identifies, segregates and disposes of substrates through lysosomal degradation therefore leading to the accumulation of AV [41-43].

To confirm and better characterize the link between GSK650394 treatment and autophagy, we first tested the expression of two specific markers of autophagy namely p62/SQSTM1 (herein p62) and LC3 in our experimental model. As a positive control we used Bafilomycin A1 (Baf A1), a known inhibitor of the autophagy flux at late stages that, as expected, increased PT-sensitivity of MDAH cells (Figs. 4d and S6b). Western blot analyses showed that GSK650394 treatment, alone or in combination with platinum, induced a clear increase in p62 levels, a change in the ratio between unlipidated (cytosolic) and lipidated (membrane bound) forms of LC3 (LC3I/LC3II ratio) and an overall increase of LC3 forms, in both MDAH and TOV21G cells comparable to the effects exerted by Baf A1 (Figs. 4d and S6c). Similar observations were made in TNBC and HNSCC cells where GSK650394 treatment also induced the increase of p62 and LC3II (Fig. S6d). Increased levels of p62 and of LC3 lipidation (LC3II) together with the accumulation of $\mathrm{AV}$, are possible markers of autophagy inhibition [42, 44, 45], overall confirming the possibility that SGK2 is a previously undisclosed regulator of autophagy. 

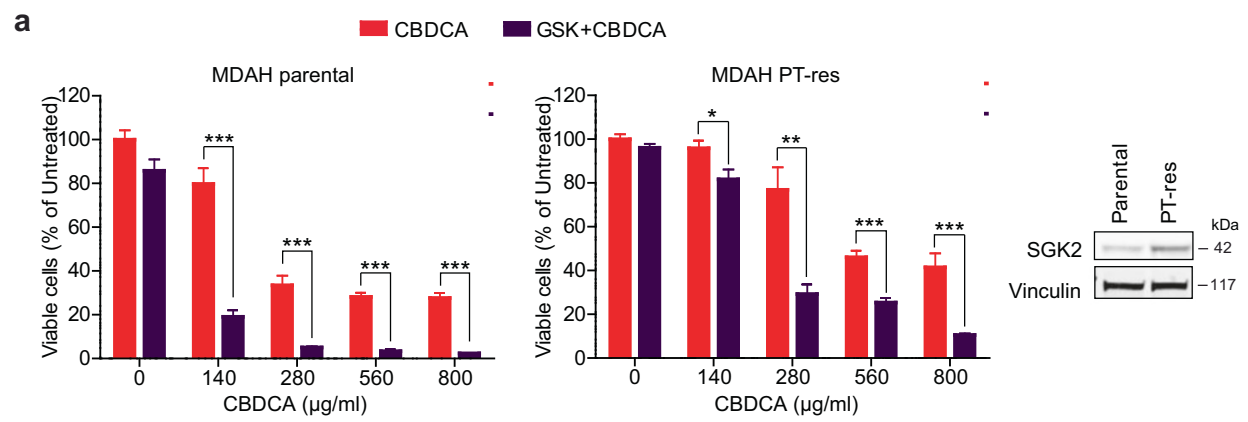

b

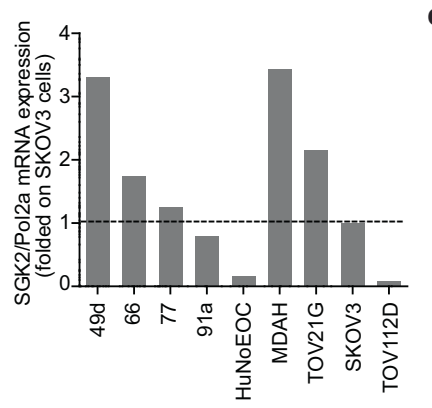

d

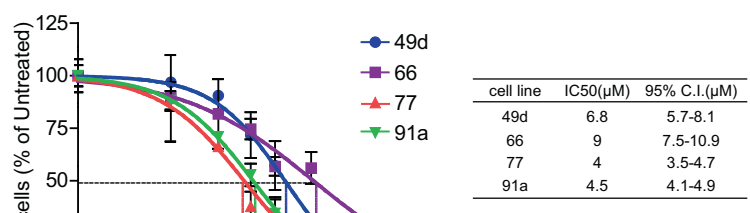

GSK GSK +CBDCA

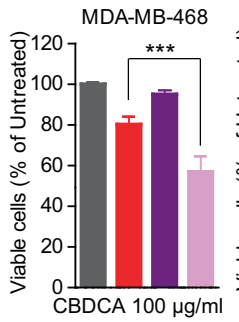

Untreated CBDCA

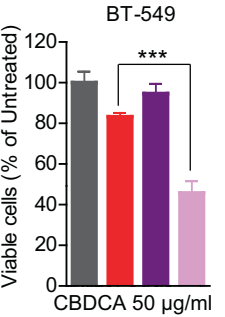

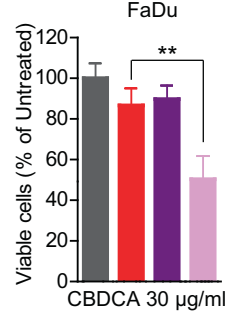
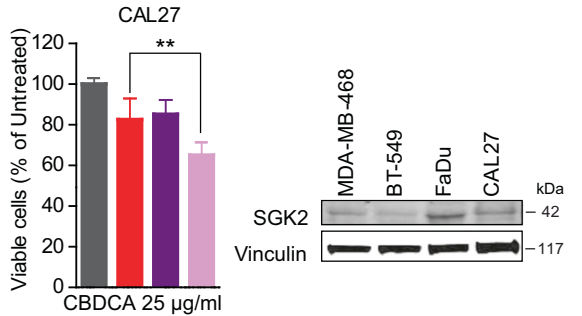

Fig. 3 SGK2 inhibition improves platinum response in platinumresistant EOC, primary EOC, head and neck squamous cell carcinoma and breast cancer cells. a Graph reporting cell viability of MDAH parental and PT-res cells treated with GSK650394 $35 \mu \mathrm{M}$ for $24 \mathrm{~h}$ (GSK), in the presence of increasing doses of CBDCA for additional $16 \mathrm{~h}$. Results are expressed as survival ratio (\%) between treated and untreated cells (set as $100 \%$ as reference). On the right, WB analysis reports the expression of SGK2. Vinculin was used as loading control. b SGK2 mRNA expression in the indicated primary and established EOC cell lines evaluated by qRT-PCR normalized to housekeeping gene POL2A and then folded on SKOV3 SGK2 expression. c Nonlinear regression analyses of cell viability assay in

\section{SGK2 inhibition alters acidification of vacuolar cellular compartment}

To better define how SGK2 down-modulation could alter the autophagic flux, we exploited a fluorescence assay using a LC3 construct tagged to mRFP-GFP tandem fluorescent proteins (ptfLC3) [46]. The use of ptfLC3 allows monitoring vesicles maturation since the EGFP protein is quenched in acidic environments (such as lysosomes and autolysosomes), whereas mRFP is more stable under acidic conditions allowing defining if autophagic vesicles are (red fluorescence) or not (red plus green = yellow fluorescence) primary EOC cells treated with increasing doses of CDDP for $16 \mathrm{~h}$. Results are expressed as percentage of viable cells respect to the untreated cells and represent the mean $( \pm \mathrm{SD})$ of three biological replicates. The table on the right reports the IC50 and the confidence interval (C.I.) of each condition. Fisher's exact test was used to calculate the global $p$ value reported in the graph. $\mathbf{d}$ Graph reporting cell viability of MDA-MB-468, BT-549 (TNBC cell lines) and FaDu and CAL27 (HNSCC cell lines) treated with GSK650394 $35 \mu$ M (GSK) and $\mathrm{CBDCA}$ as indicated. Results are expressed as survival ratio (\%) between treated and untreated cells (set as $100 \%$ as reference). On the right, WB analysis reporting the expression of SGK2. Vinculin was used as loading control. (See also Fig. S4).

fused with lysosomes [42, 46, 47]. Using this tool in MDAH cells we observed that exposure of cells to GSK650394 or SGK2 silencing inverted the ratio between red and yellow LC3 positive vesicles, here referred to as red or yellow puncta (Fig. 5a). Similarly, Baf A1 treatment that blocks lysosome acidification, also resulted in a strong increase in the number of yellow puncta. These data suggested that SGK2 inhibition could suppress autophagy at a late stage likely blocking the fusion of autophagosome to lysosome and/or autolysosome acidification.

These data were confirmed using the acridine orange (AO) staining. AO is an acidotropic dye that turns from 
a

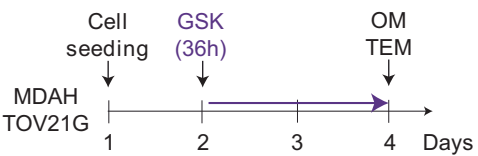

b

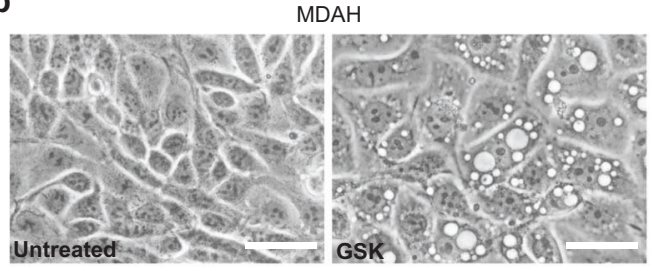

C
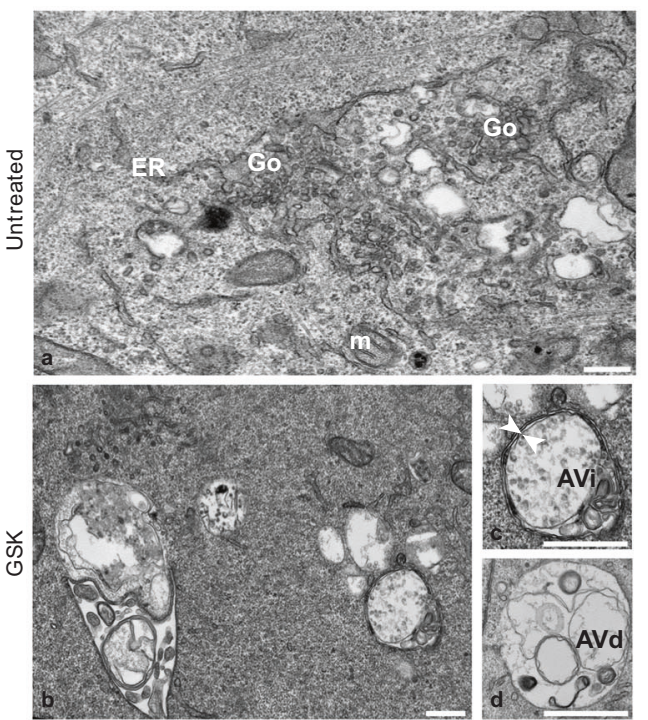

d

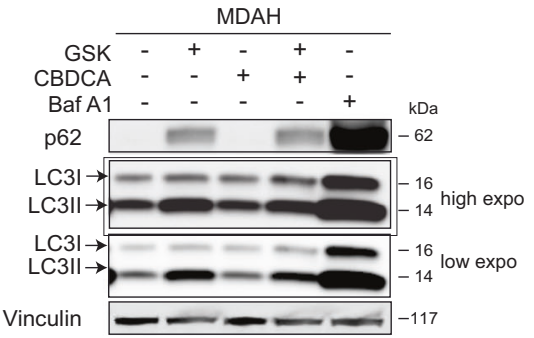

Fig. 4 SGK2 inhibition alters autophagic flux. a Experimental timeline of cells studied with optical (OM) and transmission electron microscopy (TEM). MDAH and TOV21G cells were treated with GSK650394 (GSK) $35 \mu \mathrm{M}$ for $36 \mathrm{~h}$. b Representative optical microscopy pictures of MDAH and TOV21G cells treated or not with GSK650394 (GSK) as reported in a. Cytoplasmic vacuoles are present only in GSK650394 (GSK) treated cells. Scale bars $=50 \mu \mathrm{m}$. c TEM ultrastructure analyses of MDAH (left panels) and TOV21G (right panels) cells. a and e Untreated cells; b and f GSK650394-treated cells (GSK) showing cytoplasmic vacuoles accumulation; $\mathbf{c}$ and $\mathbf{g}$

green fluorescence when located in the cytosol to a granular red fluorescence when included into acidic compartments [42, 48]. AO staining is modified by serum starvation (increased autophagy, with increased Red/Green fluorescence intensity ratio (R/G FIR)) and Baf A1 (blocked
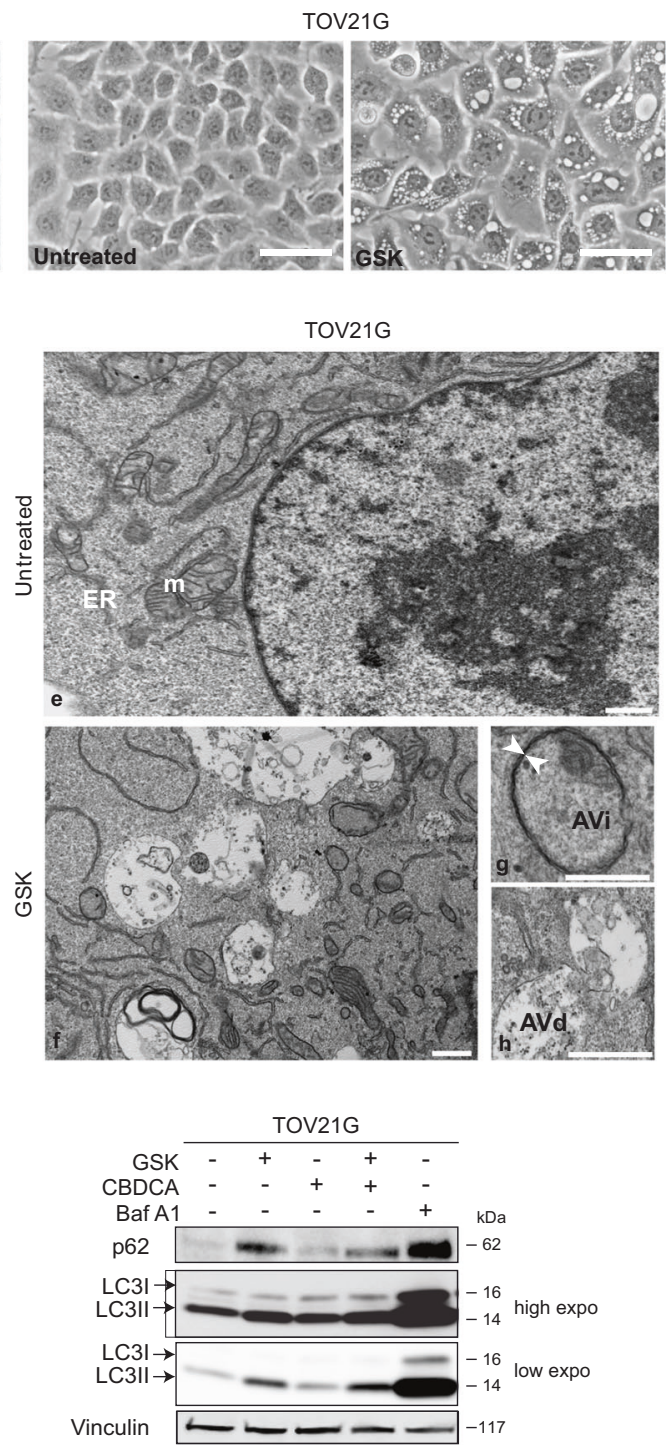

Magnification of initial autophagic vacuoles (AVi) (white arrowheads indicate double-membrane) and degradative autophagic vacuoles $(\mathrm{AVd})$ vacuoles $(\mathbf{d}$ and $\mathbf{h})$. Highlighted intracellular organelles in untreated cells are endoplasmic reticulum (ER), Mitochondrion (m) and Golgi apparatus (Go). Scale bar: $300 \mathrm{~nm}$. d Western blot analyses evaluating the expression of the autophagy markers LC3I/LC3II and p62 in MDAH and TOV21G cells, treated with GSK650394 $35 \mu \mathrm{M}$ (36 h), CBDCA $140 \mu \mathrm{g} / \mathrm{ml}(16 \mathrm{~h})$ and GSK+CBDCA (as in Fig. 2d) compared to the effect of Bafilomycin A1 (Baf A1). Vinculin was used as loading control. (See also Figs. S5 and S6).

autophagy with decreased R/G FIR). Using these two treatments as positive and negative controls, we observed that GSK650394 and Baf A1 induced a strong reduction of R/G FIR while serum starvation increased the granular R/G FIR when compared to untreated MDAH cells (Fig. 5b). 
Fig. 5 SGK2 inhibition alters acidification of vacuolar cellular compartment. a Representative pictures of confocal microscopy analyses of MDAH cells transfected with mRFP-GFP-LC3 untreated (Control), treated with GSK650394 $35 \mu \mathrm{M}$ for $36 \mathrm{~h}$ (GSK) or with Bafilomycin A1 $0.2 \mu \mathrm{M}$ for $30 \mathrm{~min}$ (Baf A1) or transduced with SGK2 shRNA (SGK2 sh2) (scale bar: $12 \mu \mathrm{m}$ ). The graph on the right reports the percentage of yellow/red LC3 puncta counted per cell (number of cells/experimental condition $=15-20)$. $\mathbf{b}$

Representative pictures of confocal microscopy analyses of MDAH cells treated or not with GSK650394 $35 \mu \mathrm{M}$ (GSK) for $36 \mathrm{~h}$ and then stained with acridine orange. Cells serumstarved for $4 \mathrm{~h}$ (Starvation) or treated with Bafilomycin A1 $0.2 \mu \mathrm{M}$ for $30 \mathrm{~min}$ (Baf A1) were used as positive and negative controls, respectively (scale bar: $18 \mu \mathrm{m}$ ). On the right, the graph reports the average red-to-green fluorescence intensity ratio (number of fields evaluated per condition $=8-10$ ) c Representative pictures of confocal microscopy analyses for the evaluation of LGALS1 (galectin-1, green) and LAMP2 (red) staining of MDAH (left) and TOV21G (right) cells treated or not with GSK650394 $35 \mu \mathrm{M}$ (GSK) or LLOMe $3 \mathrm{mM}$ (used as positive control of LMP (Lysosomal Membrane Permeabilization)) and then evaluating LGALS1 (galectin-1, green) (Scale bar: $20 \mu \mathrm{m}$ ). a
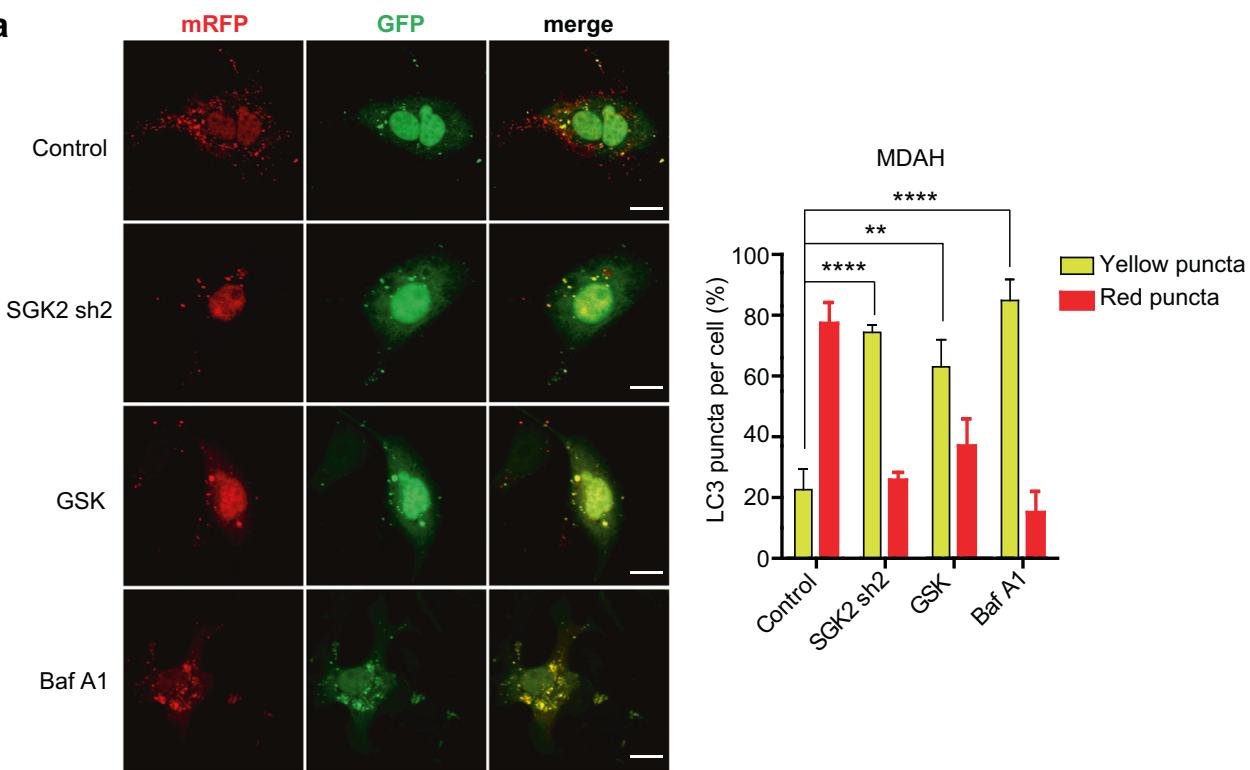

b
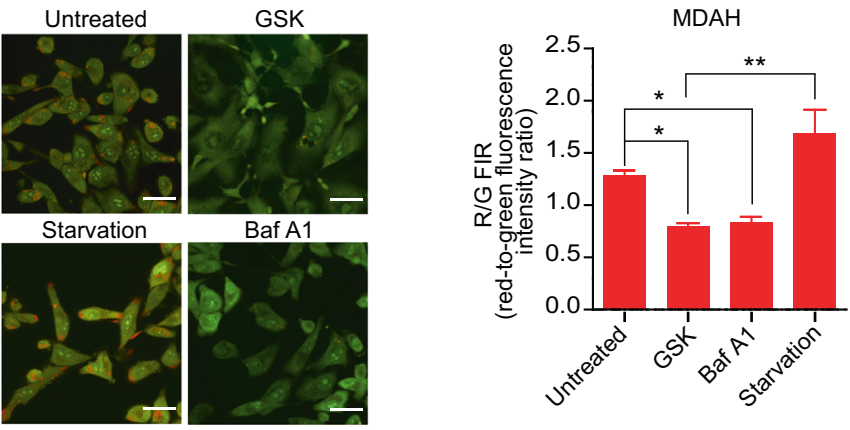

C

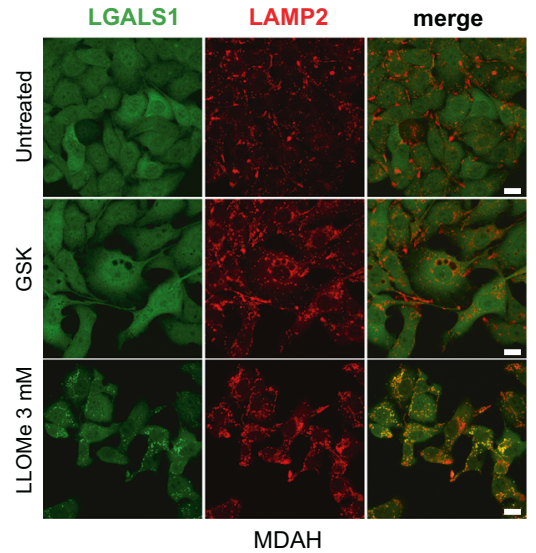

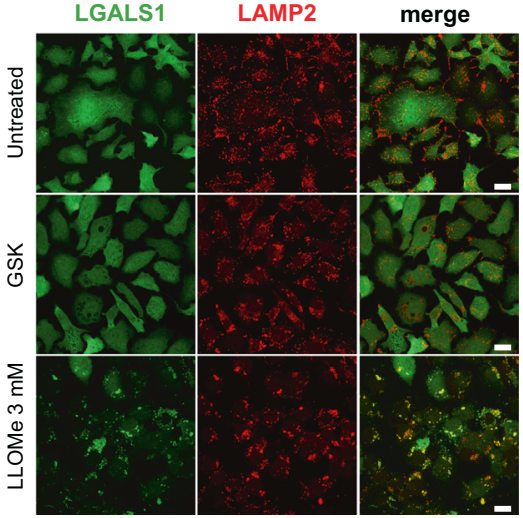

TOV21G
Since the reduction of AO R/G FIR could also be due to lysosomal membrane permeabilization (LMP) we tested if GSK650394 affected LMP rather than autophagosome maturation/acidification using the so-called galectin assay and the L-leucyl-L-leucine methyl ester (LLOMe) as positive control. LLOMe is an inducer of LMP that results in exposure of $\mathrm{N}$-acetyl-lactosamine-rich carbohydrate shield on the lysosomal membrane that therefore is bound by the translocation of LGALS1 (galectin-1). As a consequence, the LGALS1 staining changes from diffuse to punctate upon LMP induction. Data obtained in both MDAH and TOV21G showed that LLOMe but not GSK650394 induced the appearance of LGALS1 punctate staining that also colocalize with the lysosomal marker LAMP2 (Fig. 5c), overall confirming that SGK2 inhibition did not directly induce LMP.

Our data so far indicated that the inhibition of SGK2 kinase activity results in the block of autophagy at the stage 
of autophagosome/lysosome fusion and/or autolysosome acidification. To confirm these data, we stained control and GSK650394-treated cells (MDAH and TOV21G) with LAMP2 (Lysosomal Associated Membrane Protein 2) and observed a clear upregulation of LAMP2 staining in treated cells (Fig. 6a, b). LC3/LAMP2 co-staining revealed that both these molecules were present on the membrane of the large vacuoles observed under GSK treatment in both MDAH and TOV21G cells (Fig. 6c, d). Finally, co-staining of p62 with LAMP2 or SGK2 demonstrated that both LAMP2 and SGK2 co-localized on the autophagic vacuoles' membrane in GSK650394-treated cells (Fig. S7a, b). Overall, the collected data suggested that SGK2 inhibition induced the accumulation of AVd with changes in acidic cellular compartment but no effect on autophagosome/lysosome fusion or lysosome permeability.

\section{SGK2 inhibition impairs lysosomal functionality}

We next explored from a biochemical point of view if SGK2 inhibition could impact on lysosomal activity. Time-course analysis of GSK650394-treated MDAH and TOV21G cells clearly revealed that the activation of the lysosomal proteases cathepsin-D, -L and -B was impaired in a timedependent manner resulting in the accumulation of their non-active forms (Figs. 7a and S8a). The accumulation of inactive cathepsins was accompanied by the upregulation of LAMP2, p62, and LC3II (Fig. 7a). The molecular changes induced by GSK650394 were mostly recapitulated in SGK2silenced cells although the modifications were less evident (Fig. 7b). Finally, over-expression of SGK2 in OVCAR8 cells reduced the expression pattern of p62 and LC3II and increased the expression of cathepsin D (Fig. S8b).

\section{SGK2 binds and phosphorylates the V-ATPase proton pump}

All the biochemical and biological collected results (Figs. 5-7) suggested that SGK2 genetic or pharmacological inhibition impaired autolysosome acidification and degradation capacity with the consequent accumulation of late autophagic vacuoles (AVd). Since lysosome acidification depends on the activity of vacuolar $\mathrm{H}^{+}$-ATPase proton pump (V-ATPase) [49] and SGK proteins could regulate different ATPases [50, 51], we hypothesized that inhibition of SGK2 could alter the activity of this proton pump. In silico analyses predicted that SGK2 could phosphorylate several Serine and Threonine residues in the $\mathrm{V} 1 \mathrm{H}$ and V0A3 subunits which belong, respectively, to the V1 and V0 domains of V-ATPase (Fig. S8c). We explored this possibility by testing if SGK2 could interact with any of these subunits. By immunoprecipitation analysis we observed that endogenous SGK2 co-precipitated with
ATP6V1H and that this interaction was impaired by GSK650394 inhibitor treatment in a time-dependent manner (Figs. 7c and S8d). In addition, GSK650394 treatment affected the ATPase complex since it also impaired the coprecipitation of ATP6V0A3 with ATP6V1H. These observations were confirmed by over-expressing SGK2 in OVCAR8 cells where SGK2 co-precipitated with ATP6V1H and ATP6V0A3 subunits and increased the association between the two V-ATPase subunits (Fig. 7d). These evidences indicated that SGK2 interaction with VATPase could contribute directly to its activity, since the reversible assembly/disassembly of its V0 and V1 sectors represents an important regulatory mechanism of V-ATPase pump functionality $[52,53]$. Next we tried to verify if SGK2 could phosphorylate the immunoprecipitated ATP6V1H subunit using recombinant SGK2 protein in in vitro kinase assays (Fig. 7e). These assays revealed that SGK2 phosphorylated in vitro ATP6V1H subunit immunoprecipitated from both untreated and platinum treated EOC cells. Importantly, the GSK650394 inhibitor impaired the SGK2-dependent phosphorylation of ATP6V1H (Fig. 7e). It is interesting to note that SGK2 underwent a specific auto-phosphorylation (Figs. $7 \mathrm{e}$ and S8e) that, in our knowledge, was not previously described. This event was pointed out by the presence of a radioactive signal also when SGK2-GST was incubated only with $\gamma$ P32ATP in vitro while it did not phosphorylate a GST-Rb fragment used as a control (Fig. S8e).

\section{SGK2 is involved in platinum-induced autophagy response in EOC cells}

The collected data suggested that SGK2 controls autophagic flux by ensuring the correct lysosomal acidification. Autophagy has been described as a pro-survival response to chemotherapeutic drugs in cancer cells, and impairment of autophagy could represent a novel therapeutic strategy to improve the chemotherapy efficacy and/or overcome the onset of chemotherapy resistance [54-58]. To verify if autophagy was implicated in the PT-response in the used models, we first treated MDAH cells with increasing doses of CBDCA for up to $48 \mathrm{~h}$ and observed a time- and dosedependent stimulation of autophagy characterized by increased p62 clearance, LC3 I/II ratio and cathepsin D active form (Fig. 8a). Interestingly, CBDCA induction of autophagy, revealed by decreased expression of p62 in western blot analyses, was also confirmed in breast and head and neck cancer cells (Fig S9a).

We also explored autophagy and apoptosis markers 24 and $36 \mathrm{~h}$ after treatment withdrawn that represent the timing at which we observed increased cell death by the GSK650394+CBDCA (release in Fig S9b). In complete agreement with the biological data we observed that in 

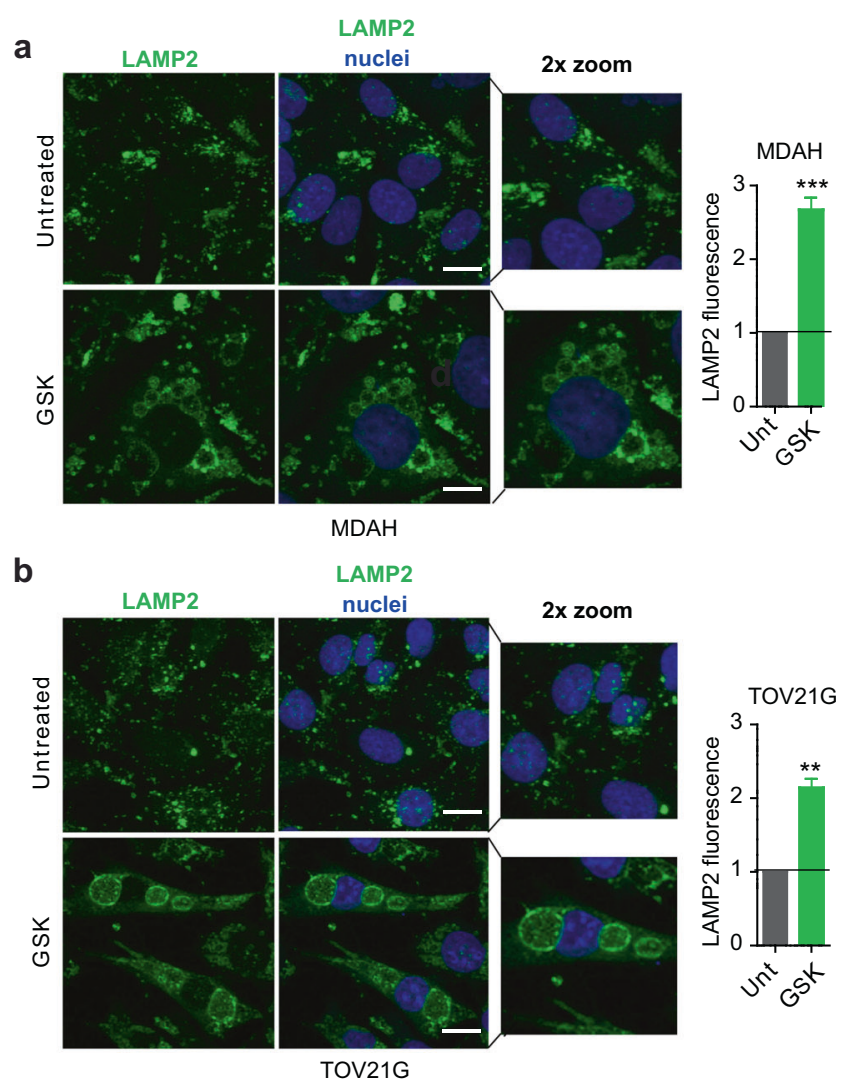

C

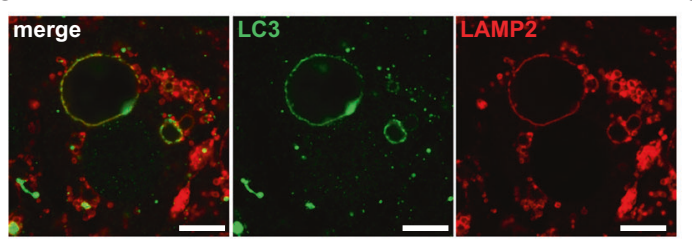

d

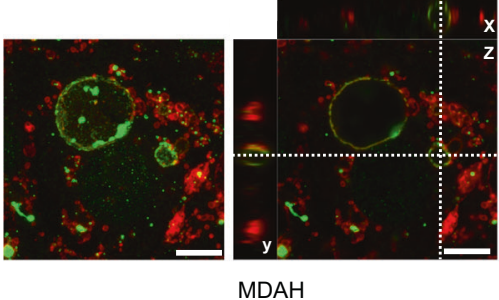

MDAH

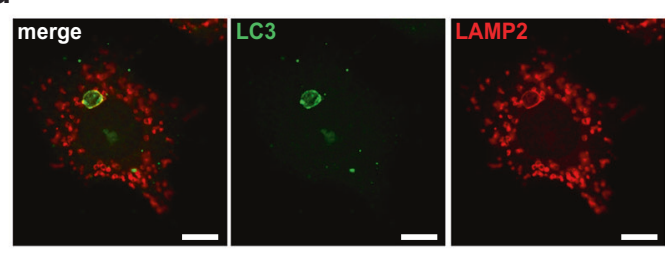

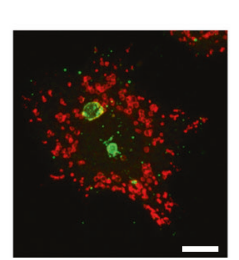

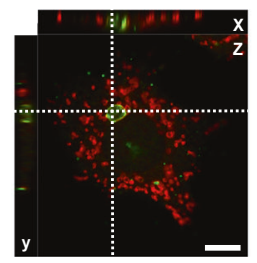

TOV21G
Fig. 6 SGK2 inhibition induces the accumulation of autophagolysosomes. Representative pictures of confocal microscopy immunofluorescence analyses evaluating the expression and localization of LAMP2 in untreated or GSK650394 (GSK)-treated MDAH a and TOV21G b cells. A zoomed area is reported for each condition. Nuclei were stained with propidium iodide and pseudo colored in blue (scale bars: $9 \mu \mathrm{m}$ ). Graphs on the right report the average LAMP2 fluorescence intensity per field (average number of cells evaluated per condition $=90$ cells). Results are expressed as fold of green fluorescence respect to untreated. Representative pictures of confocal microscopy

cells treated with GSK650394+CBDCA modulation of autophagy preceded the appearance of apoptotic markers (i.e., cleaved PARP-1 and caspase-9), suggesting that the combination treatment induced increased cell death by immunofluorescence analyses evaluating LC3 (green) and LAMP2 (red) expression and localization in MDAH $\mathbf{c}$ and TOV21G d cells treated with GSK650394. Upper panel shows a representative best focus plane with single and merged channels, as indicated. Lower panel shows the maximum projection (left) and the xyz projections (right) showing the co-immunostaining of LC3 and LAMP2 in a vacuole after GSK650394 treatment. Scale bar:10 $\mu \mathrm{m}$. In the figure data are mean \pm SD. Significance was calculated using two-tailed, unpaired Student's $t$ test. ${ }^{* *} p<0.001,{ }^{* *} p<0.01$. (See also Fig. S7).

impairing autophagy with a consequent induction of apoptosis.

To better dissect molecular events, we observed that stable over-expression of SGK2 in OVCAR8 cells that 
a

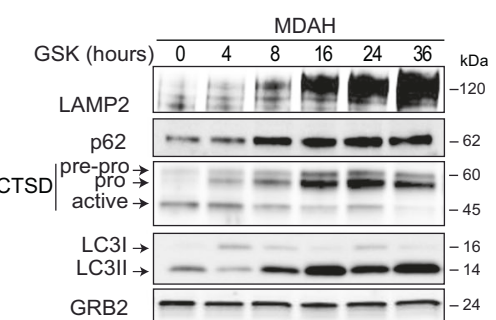

b

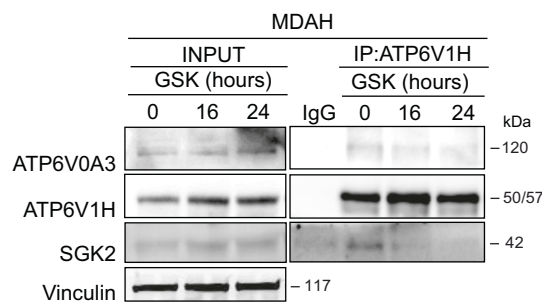

C

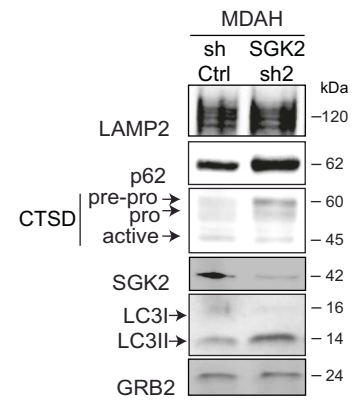

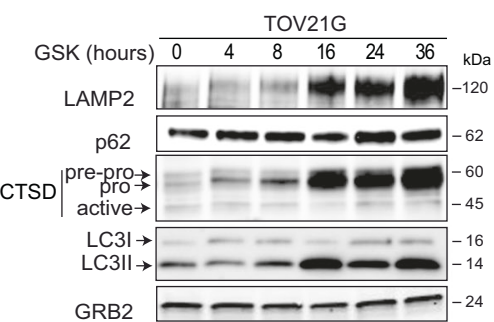

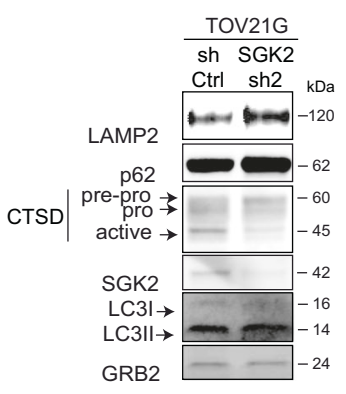

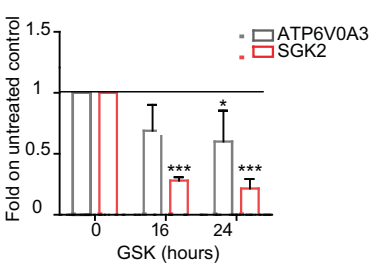

d

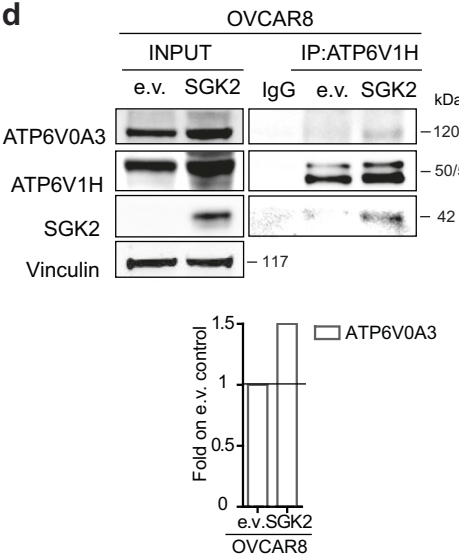

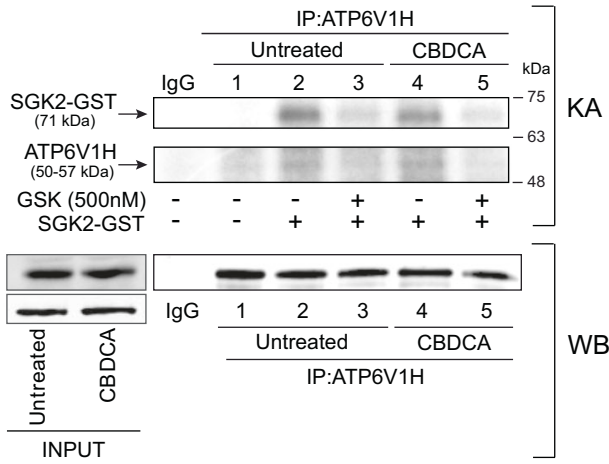

resulted in increased PT-resistance (Fig. 1e) also increased autophagy as demonstrated by the higher clearance of p62, inversion of LC3 I/II ratio and by a marked increase in active cathepsin D (Fig. 8b).

Next, by overexpressing SGK2 WT, KD and DN constructs in TOV21G we observed that the WT protein induced a rapid down modulation of p62 and LC3 already at the low dose of $100 \mu \mathrm{g} / \mathrm{ml} \mathrm{CBDCA}$ while the DN mutant induced a persistent expression of p62 likely due to autophagy blockade (Fig. 8c). As expected, the expression of SGK2 KD did not induce any significant difference respect to TOV21G parental cells transfected with empty vector (Fig. 8c).
The data collected so far demonstrated that in EOC cells CBDCA treatment induced autophagy as a surviving mechanism sustained by SGK2 kinase activity. Accordingly, by treating MDAH cells with increasing doses of CBDCA in presence or not of GSK we observed a reversion of CBDCA-induced autophagy in cells treated with GSK650394+CBDCA. This reversion was evidenced by both delayed p62 clearance and inversion ratio LC3I/II (Fig. S10a).

We next asked if induction of autophagy was commonly induced by other chemotherapy used in the treatment of EOC and tested the effects of Taxol on p62 and LC3 
Fig. 7 SGK2 inhibition causes autophagy blockade altering activation of lysosomal cathepsins. WB analyses of LAMP2, p62, Cathepsin D (CTSD) and LC3I/LC3II in cell lysates from MDAH and TOV21G cells treated with GSK650394 $35 \mu \mathrm{M}$ (GSK) for the indicated time a or silenced for SGK2 expression b. GRB2 was used as loading control. c Co-immunoprecipitation (IP) analysis of endogenous ATP6V1H with SGK2 and ATP6V0A3 in MDAH cells untreated or treated with GSK650394 $35 \mu \mathrm{M}$ (GSK) for 16 and $24 \mathrm{~h}$. Cell lysates immunoprecipitated with ATP6V1H were probed for the expression of ATP6V1H, SGK2, and ATP6V0A3. The expression of the three proteins in the corresponding lysates (INPUT) is reported on the left. The right graph shows the quantification of SGK2 and ATP6V0A3 bound to ATP6V1H in GSK treated cells expressed as fold respect to untreated cells and represents the mean \pm SD of three independent experiments. Significance was calculated using two-tailed, unpaired Student's $t$ test. $* * * p<0.001, * p<0.05$. d Co-immunoprecipitation (IP) analysis of endogenous ATP6V1H with SGK2 and ATP6V0A3 in OVCAR8 cells stably overexpressing SGK2. Cell lysates immunoprecipitated with ATP6V1H were probed for the expression of ATP6V1H, SGK2 and ATP6V0A3. The expression of the three proteins in the corresponding lysates (INPUT) is reported. The lower graph shows the quantification of ATP6V0A3 bound to ATP6V1H in SGK2 overexpressing cells expressed as fold respect to cells transfected with the empty vector (e.v.). e In vitro kinase assay (upper panel) using SGK2 active recombinant protein incubated with endogenous ATP6V1H immunoprecipitated from untreated and CBDCAtreated MDAH cells $(500 \mu \mathrm{g} / \mathrm{ml} 16 \mathrm{~h})$ (lower panel). Western blot analyses of $1 / 10$ of the immunoprecipitated ATP6V1H protein used in each line (1-5) and the corresponding lysates (INPUT) are reported (lower panel). Vinculin was used as loading control in c-e. IgG indicates a lysate IP with unrelated antibody. (See also Fig. S8).

expression in MDAH cells. Dose and time response curve showed that Taxol did not elicit any modification in their expression over a $36 \mathrm{~h}$ period (Fig. S10b). Accordingly, SGK2 inhibition by GSK650394 did not affect Taxol induced cell death in MDAH cells (Fig. S10c), overall supporting the hypothesis that SGK2 kinase activity protects cancer cells from PT-induced death by regulating autophagy.

Finally, by interrogating the Oncomine $[59,60]$ (http://www.oncomine.org) datasets we found that SGK2 is not only overexpressed but also amplified in a subset of EOC compared to normal tissue (Fig. S11a-c), suggesting that it could play a role in the human pathology.

\section{Discussion}

Starting from an unbiased loss-of-function screening that identified SGK2 as a druggable modulator of PT sensitivity in EOC cells, we characterized the role of this under-studied kinase in the control of autophagy, an escaping strategy activated by cancer cells to survive under PT treatment. We demonstrated that genetic or pharmacological inhibition of SGK2 could block the induction of the autophagic process stimulated by PT, eventually increasing PT-induced cell death, in vitro.
It is known that autophagic responses in cancer cells operate in a highly dynamic network that affects complex processes going beyond intracellular homeostasis maintenance [57]. In this sense, the autophagic network plays paradoxical context-dependent roles being involved in tumor suppression and tumor promotion [58, 61]. Several evidences pointed out a potential role of autophagy in the ability of cancers to develop resistance to chemotherapy, suggesting a major impact of autophagy in the response to treatment $[62,63]$. Here we report that in EOC cells chemotherapy with diverse mechanisms of action (CBDCA vs Taxol) differently impacted on autophagy induction, further confirming the context-dependent role of autophagy in cancer.

Autophagy is a highly conserved dynamic catabolic process that involves the formation of double membrane vesicles known as autophagosomes that engulf cellular proteins and organelles for delivery to the lysosome. When the autophagosome fuses with the lysosome, the content is degraded and macromolecular precursors are recycled or used to fuel metabolic pathways [55]. The adapter protein p62, which targets specific substrates to autophagosomes, and LC3II are degraded along with other cargo proteins and can be used as a measure of autophagic flux. The appearance of more autophagic elements (i.e., autophagosomes or autolysosomes) does not necessarily mean more autophagy. In fact, in many cases autophagosomes may accumulate due to a block in trafficking to lysosomes without any change in autophagosome biogenesis, whereas an increase in autolysosomes may indicate a reduction in degradation activity. In this sense, lysosomal digestion is a stage of autophagy and analyzing its proficiency is a fundamental part of the evaluation of autophagic flux or complete autophagy [42]. Lysosomes are characterized by highly acidic $\mathrm{pH}$ that provides optimal condition for the activity of its proteolytic enzymes like cathepsins [49]. Lysosomal $\mathrm{pH}$ gradient is mainly generated and maintained by V-ATPase, a multisubunit protein complex that hydrolyzes ATP to pump protons into the lysosome [52, 53].

In this context our data suggest that SGK2 genetic or pharmacological inhibition induces an accumulation of autophagosomes, in particular of giant autolysosomes, that was accompanied by an accumulation of inactive cathepsins [64]. We propose that SGK2 kinase activity has a role in the activation of V-ATPase favoring the V0V1 sectors assembly of this proton pump, likely via phosphorylation of the ATP6V1H subunit. We observed that SGK2 is able to phosphorylate ATP6V1H in in vitro kinase assays, (Fig. 7c) and, hypothetically, that this could also happen in a cellular context since GSK650394 treatment on MDAH cells impaired SGK2-ATP6V1H/V0A3 interaction. However, further experiments should confirm this possibility. Our evidences also support the possibility that SGK2 inhibition 
Fig. 8 Platinum treatment stimulates autophagy in EOC cells. WB analyses of p62, Cathepsin D (CTSD) and LC3I/ LC3II expression in MDAH cells a or in control and SGK2overexpressing OVCAR8 cells b treated with increasing doses of CBDCA for the indicated times. c WB analysis of autophagy markers in TOV21G cells overexpressing SGK2 WT, DN and KD mutants treated as in a and $\mathbf{b}$ for $16 \mathrm{~h}$. GRB2 $\mathbf{a}$ or Vinculin $\mathbf{b}$, $\mathbf{c}$ were used as loading control. d Schematic representation of the possible role of SGK2 role in the regulation of platinum sensitivity of EOC cells. Platinum treatment induces an autophagic response as a prosurvival pathway in which SGK2 acts as an autophagy stimulator. In SGK2 expressing cells, SGK2 by interacting with the lysosomal V-ATPase proton pump, ensures the maintenance of the correct acid $\mathrm{pH}$ necessary for the activation/maturation of lysosomal enzymes (left panel). Silencing or chemical inhibition of SGK2 results in V-ATPase disassembly, impaired lysosomal degradation capacity and the consequent inhibition of autophagic flux with accumulation of giant autophagolysosomes (depicted inside the cell, right panel). Autophagy inhibition eventually results in an increased EOC cells sensitivity to platinum treatment and enhanced cell death (right panel).

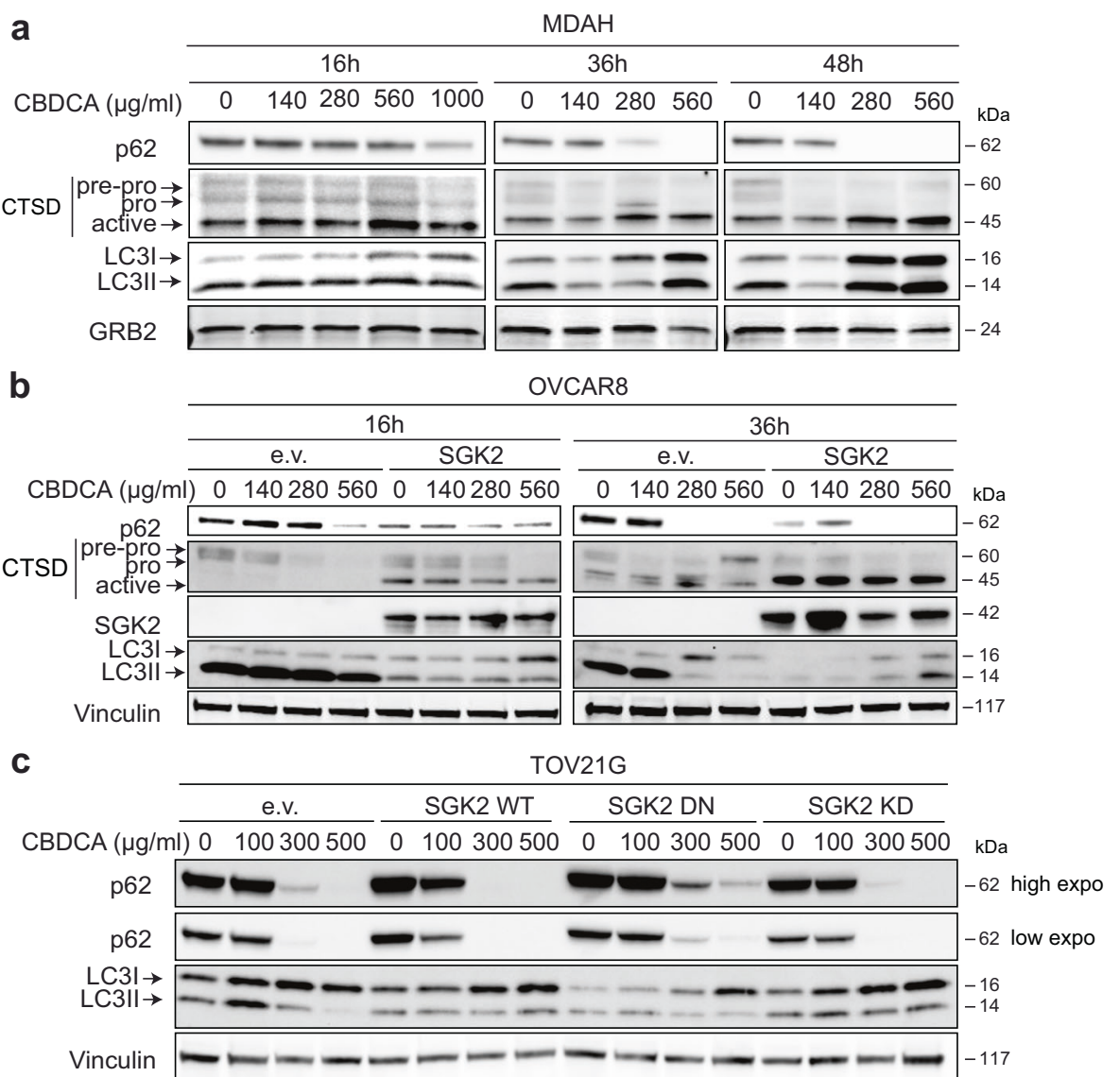

d

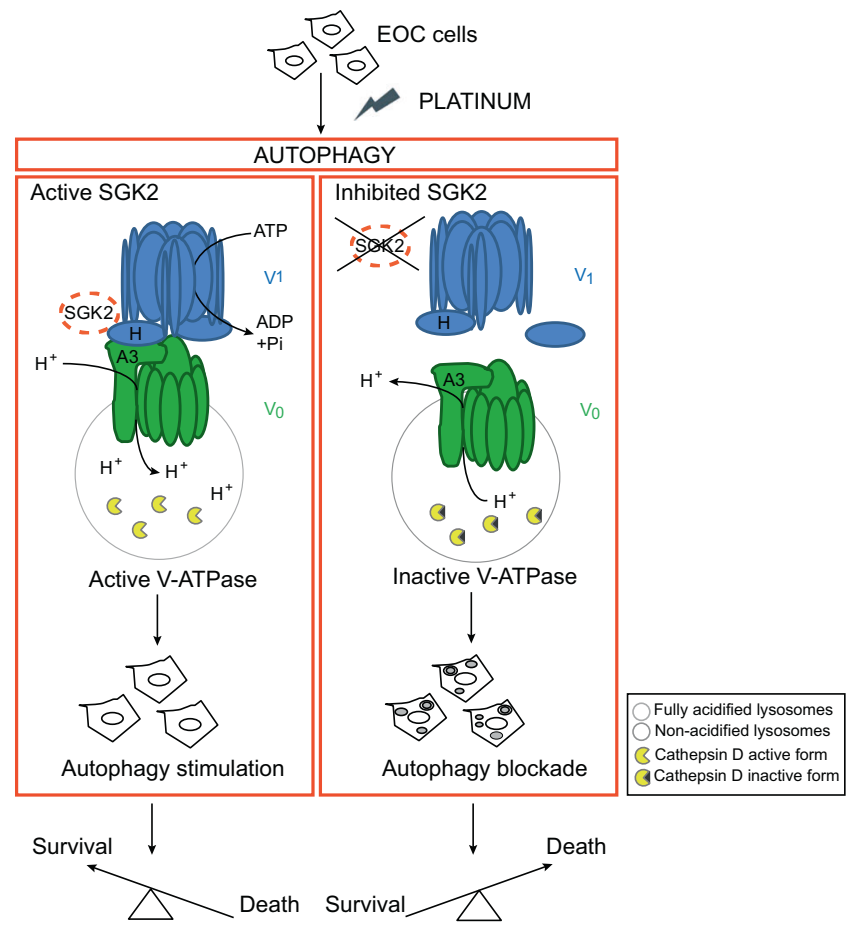

subsequent to SGK2 inhibition, then reduces lysosomal degradation capacity with the consequent accumulation of autolysosome and resulting inhibition of the autophagic flux promotes ATPase disassembly, reducing its activity in maintaining acidic lysosomal $\mathrm{pH}$ for optimal proteases maturation/activity. The impaired activation of cathepsins 
(Fig. 8d). It is worth noting that pharmacological inhibition of SGK2 with GSK650394 better blocks the autophagic flux than the silencing of the gene. This partial discrepancy could be due to the incomplete knock out of the SGK2 protein achievable with the use of the shRNAs technology or possible off target effect of the GSK650394 molecule. Future work with different molecular approaches could help in dissecting these two possibilities.

In line with our results, recent works reported that silencing different subunits of V-ATPase proton pump enhances cytotoxicity of paclitaxel and platinum drugs in cervical and ovarian cancer, respectively [65, 66]. These evidences supported the idea of new anticancer strategies based on drug repositioning of proton pumps inhibitors [67].

It is interesting to note that although SGK2 inhibition transiently blocks autophagy also in untreated and Taxoltreated cells, this block did not result in toxic effects for normal or cancer cells, therefore supporting a specific role for SGK2 inhibition in the response to PT-induced death. However, we would like to point out that SGK2, like the other members of its family, could be directly involved in the regulation of other cellular pathways like cell proliferation and apoptosis that we did not investigate here and that could also contribute to its regulation of PT-sensitivity.

Overall, we report here a new role of SGK2 kinase in the control of PT-induced cell death in different types of cancer by the inhibition of autophagy. This role seems not be shared by the two other members of the SGK family in EOC cells, although more studies, likely involving the use of CRISPR technology to knock out the different members of the family, are necessary to definitely confirm this observation. Being SGK2 a druggable kinase, amplified in a subset of EOC, for which inhibitors are already available our findings could have also an immediate translational relevance to improve the treatment and/or the quality of life of patients with PT-treatable, SGK2-expressing cancers.

\section{Materials and methods}

\section{Study approval}

Our institutional Biobank has collected samples and obtained informed consent from all patients. The Internal Review Board approved this study (\#IRB-06/2011 and IRB-05-2014).

\section{Cell culture}

MDAH-2774, SKOV3, TOV112D, TOV21G, OV90, MDAH-MB-468, BT-549, FaDu, CAL27 and 293T17 cells were purchased from ATCC; OVCAR8 from NCI; Immortalized Human Ovarian Epithelial cells (HuNoEOC) from ABM; COV318 from ECACC; 293FT from Invitrogen. Fresh tumor specimens were used to generate primary EOC cultures.

\section{Reagents}

Carboplatin (CBDCA), Cisplatin (CDDP), and Taxol (TEVA Italia) were used for in vitro experiments. GSK650394 is a SGK1/SGK2 kinase inhibitor purchased from Tocris Bioscience (3572). Bafilomycin A and Acridine Orange were purchased from Sigma-Aldrich Co and Lleucyl-L-leucine methyl ester (LLOMe) was purchased from Santa Cruz (Table S1).

\section{Plasmids and constructs}

pDONR223 SGK2 was a gift from William Hahn \& David Root (Addgene plasmid \#23378) and it was used to generate the pEGFP SGK2 vector, using pEGFP-C1 vector (Clontech), and pLPC SGK2 vector, using the pLPC vector gently provided by Dr. R. Maestro (CRO Aviano). Sitedirected mutagenesis was used to generate SGK2 DN (SGK2 T193A/S356A) mutant, SGK2 CA (SGK2 S356D) mutant and SGK2 Kinase Dead (KD) (SGK2 K64M) (primers listed in Table S1) with QuikChange Site-Directed Mutagenesis Kit (Agilent). mRFP-EGFP-LC3 was a gift from Tamotsu Yoshimori (Addgene plasmid \# 21074). pLKO shRNAs were purchased from Sigma-Aldrich Co and they are listed in Table S1.

\section{Cell viability assays}

Cells were seeded in 96-well culture plates and, after $72 \mathrm{~h}$ from transduction or $24 \mathrm{~h}$ from inhibitor treatment, cells were treated with normal growth medium containing vehicle or CBDCA/CDDP for $16 \mathrm{~h}$ at the indicated concentrations. Cell viability was evaluated $24 \mathrm{~h}$ after the end of treatment using CellTiter 96 AQueous cell proliferation assay kit (Promega). These assays were performed three times with three biological replicates for each experimental condition. Data is depicted in some experiments as Abs value at $492 \mathrm{~nm}$, in others as percentage of viable cells respect to untreated cells (set as $100 \%$ as reference) as described in figure legends. In all cases, data represent the mean $\pm \mathrm{SD}$.

\section{Preparation of cell lysates, immunoblotting, and immunoprecipitation}

Cell lysates were prepared using cold RIPA lysis buffer (150 mM NaCl, $50 \mathrm{mM}$ Tris $\mathrm{HCl}$ [ph8], 1\% Igepal, 0,5\% sodium deoxycholate, $0,1 \%$ SDS) plus a protease inhibitor cocktail (Complete, Roche), $1 \mathrm{mM}$ sodium orthovanadate, and $1 \mathrm{mM}$ dithiothreitol as previously reported [68]. Protein 
concentrations were determined using the Bio-Rad protein assay (Bio-Rad). For immunoblotting, equal concentrations of protein samples $(60 \mu \mathrm{g})$ were separated by $4-20 \%$ SDSPAGE (Criterion precast gel; Bio- Rad) and transferred to nitrocellulose (Hybond C; Amersham) or PVDF (Bio-Rad) membranes. Immunoprecipitations were performed using $1 \mathrm{mg}$ of cell lysate in HNTG buffer (20 mM HEPES, $150 \mathrm{mM} \mathrm{NaCl}, 10 \%$ glycerol, $0.1 \%$ Triton X-100) supplemented with protease inhibitors plus $2 \mu \mathrm{g}$ of the indicated specific primary antibody and incubating overnight at $4{ }^{\circ} \mathrm{C}$. The immunocomplexes were captured by incubation with protein $\mathrm{A}$ and $\mathrm{G}$ beads (GE Healthcare) for $2 \mathrm{~h}$ at $4{ }^{\circ} \mathrm{C}$, washed with HNTG buffer, eluted in $3 \mathrm{X}$ sample buffer, and separated on SDS-PAGE for western blot analysis. Immunoblotting was performed using the following primary antibodies: rabbit monoclonal anti-SGK2 (1:500), rabbit monoclonal anti-SGK3 (1:500), rabbit monoclonal LC3B (1:500), rabbit polyclonal anti-Caspase 9 (1:500) from Cell Signaling Technology and rabbit polyclonal LC3B (1:10000) from Novus Biological; mouse monoclonal antiVinculin (1:1000), mouse monoclonal anti-LAMP2 (1:200), mouse monoclonal anti-SGK1 (1:200), mouse monoclonal anti-V-ATPase H (ATP6V1H) (1:200), purchased from Santa Cruz Biotechnology; mouse polyclonal anti-SGK2 (used for IP) and mouse monoclonal anti-Cathepsin D (1:500) from Sigma Aldrich Co; rabbit monoclonal anti-p62 (1:20000) and rabbit polyclonal anti-TCIRG1/ATP6V0A3 (1:500) from Abcam; rabbit polyclonal anti-Cathepsin $\mathrm{L}$ (1:500) and rabbit polyclonal anti-Cathepsin B (1:500) from Elabscience; mouse monoclonal anti-GST (1.500) from BD Biosciences; mouse anti-GRB2 (1:500) from Transduction Lab; mouse monoclonal anti-GFP (1:500) from Roche. Antibodies were visualized with appropriate horseradish peroxidase-conjugated secondary antibodies (GE Healthcare) for ECL detection (Bio-Rad) or Alexa-conjugated secondary antibodies (Invitrogen) for Odyssey infrared detection (LI-COR Biosciences).

\section{In vitro kinase assay}

In vitro kinase assays were performed by incubating SGK2 active kinase (Signal Chem) with endogenous ATP6V1H immunoprecipitated from untreated and CBDCA-treated MDAH cells (CBDCA $500 \mu \mathrm{g} / \mathrm{ml}, 16 \mathrm{~h}$ ) or Rb1-GST recombinant protein, as substrates, for $30 \mathrm{~min}$ at $30^{\circ} \mathrm{C}$ in the presence of $1.5 \mu \mathrm{Ci}$ of $\gamma^{32} \mathrm{P}$-ATP (PerkinElmer), as previously described [69].

\section{qRT-PCR analyses}

qRT-PCR analyses were performed as previously described [68]. Briefly, total RNA was extracted using Trizol reagent (Invitrogen), quantified using NanoDrop (Thermo Fisher
Scientific Inc., USA), and retro-transcribed using the AMV reverse transcriptase (Promega). cDNAs were amplified using SsoFast EvaGreen Supermix (Biorad) and CFX96 ${ }^{\mathrm{TM}}$ Real-Time PCR Detection System (Bio-Rad). SGK2 expression was normalized using SGK2/housekeeping gene as SQ mean ratio. The SQ (Starting Quantity) was calculated using $\mathrm{CFX} 96^{\mathrm{TM}}$ software (Bio-Rad).

\section{Immunofluorescence}

For immunofluorescence, cells plated on coverslips and fixed in PBS 4\% paraformaldehyde (PFA) were stained with primary antibodies, such as SGK2 (1:400) and LAMP2 (1:200) (Santa Cruz Biotechnologies), LC3B (1:200) (Cell Signaling), LGALS1 (1:1000) (Abcam) and p62 (1:200) (Abcam). Propidium iodide $(5 \mu \mathrm{g} / \mathrm{ml})$ was used for nuclear staining as reported $[38,70]$. For acridine orange staining, cells were incubated with acridine orange solution (SigmaAldrich Co) for $30 \mathrm{~min}$ at $37^{\circ} \mathrm{C}$ before image acquisition. Cells were treated with Bafilomycin A $0.2 \mu \mathrm{M}$ for $30 \mathrm{~min}$ at $37{ }^{\circ} \mathrm{C}$ for controls. Stained cells were analyzed using a confocal laser-scanning microscope (TCS SP8 Leica). Fluorescence intensity and protein co-localization were studied using the Volocity ${ }^{\circledR}$ software (PerkinElmer) (See also Supplementary Materials and methods).

\section{Statistical analyses}

Statistical significance $(p<0.05)$, means, standard deviation, $95 \%$ confidence intervals (CIs) were determined by using GraphPad PRISM software (version 6.0) using the most appropriate test, as specified in each figure. Statistical significance was indicated with: $* p<0.05, * * p<0.01$, $* * * p<0.001, * * * * p<0.0001$

SGK2 expression in available ovarian cancer dataset is reported from Oncomine website (http://www.oncomine. org), statistical analysis is described in each figure [59, 60].

Acknowledgements We thank Dr William Hahn and David Root for SGK2 vector (through Addgene), Dr Tamotsu Yoshimori for ptfLC3 vectors (through Addgene), Dr Roberta Maestro for $\mathrm{pLPC}$ vector and Laura Cesaratto and Sara Benevol for technical support. We are grateful to all members of our lab for helpful and stimulating discussions. This work was supported by grants from Ministero della Salute (RF-2010-2309704 and RF-2016-02361040), Regione Friuli Venezia Giulia (Tichep Grant) and $5 \times 1000$ CRO to GB; grants from $5 \times 1000$ CRO Intramural Young Investigator and from Ministero della Salute (RF-2016-02361041) to MSo. IS was supported by an AIRC biannual Fellowship (\# 18171). The funders had no role in study design, data collection and analysis, decision to publish, or preparation of the manuscript.

Author contributions MSc and GB designed the experiments. VR, IL, and IP performed most of the experiments. ADA and MSc performed the functional genomic screening. JP and DS performed the statistical analysis of high throughput screening. PSa performed T.E.M. 
analyses. PSp performed confocal microscopy analyses. ADA, MSo, IS, SDA, IP, MC, and SL contributed to the experimental work. MSo and IL selected resistant cells. IP prepared most of figures and graphs. GB and MSc analyzed the data and wrote the manuscript. BB and PSp contributed to analyze the data, drafting, editing, and critically revising the article. All authors read and approved the manuscript.

\section{Compliance with ethical standards}

Conflict of interest The authors declare that they have no conflict of interest.

Publisher's note Springer Nature remains neutral with regard to jurisdictional claims in published maps and institutional affiliations.

Open Access This article is licensed under a Creative Commons Attribution 4.0 International License, which permits use, sharing, adaptation, distribution and reproduction in any medium or format, as long as you give appropriate credit to the original author(s) and the source, provide a link to the Creative Commons license, and indicate if changes were made. The images or other third party material in this article are included in the article's Creative Commons license, unless indicated otherwise in a credit line to the material. If material is not included in the article's Creative Commons license and your intended use is not permitted by statutory regulation or exceeds the permitted use, you will need to obtain permission directly from the copyright holder. To view a copy of this license, visit http://creativecommons. org/licenses/by/4.0/.

\section{References}

1. Deans AJ, West SC. DNA interstrand crosslink repair and cancer. Nat Rev Cancer. 2011;11:467-80.

2. Kang X, Xiao H-H, Song H-Q, Jing X-B, Yan L-S, Qi R-G. Advances in drug delivery system for platinum agents based combination therapy. Cancer Biol Med. 2015;12:362-74.

3. Holmes D. The problem with platinum. Nature. 2015;527: S218-219.

4. Holmes D. Ovarian cancer: beyond resistance. Nature. 2015;527: S217.

5. Imai H, Kaira K, Mori K, Ono A, Akamatsu H, Taira T, et al. Comparison of platinum combination re-challenge therapy and docetaxel monotherapy in non-small cell lung cancer patients previously treated with platinum-based chemoradiotherapy. SpringerPlus. 2015;4:152.

6. Lo Russo G, Macerelli M, Platania M, Zilembo N, Vitali M, Signorelli D, et al. Small-cell lung cancer: clinical management and unmet needs new perspectives for an old problem. Curr Drug Targets. 2017;18:341-62.

7. Machiels J-PH, Licitra LF, Haddad RI, Tahara M, Cohen EE. Rationale and design of LUX-Head \& Neck 1: a randomised, Phase III trial of afatinib versus methotrexate in patients with recurrent and/or metastatic head and neck squamous cell carcinoma who progressed after platinum-based therapy. BMC Cancer. 2014;14:473

8. Massarelli E, Lin H, Ginsberg LE, Tran HT, Lee JJ, Canales JR, et al. Phase II trial of everolimus and erlotinib in patients with platinum-resistant recurrent and/or metastatic head and neck squamous cell carcinoma. Ann Oncol J Eur Soc Med Oncol. 2015;26:1476-80.

9. Bast RC, Hennessy B, Mills GB. The biology of ovarian cancer: new opportunities for translation. Nat Rev Cancer. 2009;9:415-28.

10. Jayson GC, Kohn EC, Kitchener HC, Ledermann JA. Ovarian cancer. Lancet Lond Engl. 2014;384:1376-88.
11. Cancer Genome Atlas Research Network. Integrated genomic analyses of ovarian carcinoma. Nature. 2011;474:609-15.

12. Patch A-M, Christie EL, Etemadmoghadam D, Garsed DW, George J, Fereday S, et al. Whole-genome characterization of chemoresistant ovarian cancer. Nature. 2015;521:489-94.

13. Vaughan S, Coward JI, Bast RC, Berchuck A, Berek JS, Brenton $\mathrm{JD}$, et al. Rethinking ovarian cancer: recommendations for improving outcomes. Nat Rev Cancer. 2011;11:719-25.

14. Galluzzi L, Vitale I, Michels J, Brenner C, Szabadkai G, HarelBellan A, et al. Systems biology of cisplatin resistance: past, present and future. Cell Death Dis. 2014;5:e1257.

15. Gatti L, Cassinelli G, Zaffaroni N, Lanzi C, Perego P. New mechanisms for old drugs: insights into DNA-unrelated effects of platinum compounds and drug resistance determinants. Drug Resist Updat Rev Comment Antimicrob Anticancer Chemother. 2015;20:1-11.

16. Dikic I, Elazar Z. Mechanism and medical implications of mammalian autophagy. Nat Rev Mol Cell Biol. 2018;19:349-64.

17. Galluzzi L, Senovilla L, Vitale I, Michels J, Martins I, Kepp O, et al. Molecular mechanisms of cisplatin resistance. Oncogene. 2012;31:1869-83.

18. Kimmelman AC, White E. Autophagy and tumor metabolism. Cell Metab. 2017;25:1037-43.

19. Liu D, Yang Y, Liu Q, Wang J. Inhibition of autophagy by 3-MA potentiates cisplatin-induced apoptosis in esophageal squamous cell carcinoma cells. Med Oncol Northwood Lond Engl. 2011;28:105-11.

20. Ren J-H, He W-S, Nong L, Zhu Q-Y, Hu K, Zhang R-G, et al. Acquired cisplatin resistance in human lung adenocarcinoma cells is associated with enhanced autophagy. Cancer Biother Radiopharm. 2010;25:75-80.

21. Yu H, Su J, Xu Y, Kang J, Li H, Zhang L, et al. p62/SQSTM1 involved in cisplatin resistance in human ovarian cancer cells by clearing ubiquitinated proteins. Eur J Cancer Oxf Engl 1990. 2011;47:1585-94.

22. Arencibia JM, Pastor-Flores D, Bauer AF, Schulze JO, Biondi RM. AGC protein kinases: from structural mechanism of regulation to allosteric drug development for the treatment of human diseases. Biochim Biophys Acta. 2013;1834:1302-21.

23. Pearce LR, Komander D, Alessi DR. The nuts and bolts of AGC protein kinases. Nat Rev Mol Cell Biol. 2010;11:9-22.

24. Kobayashi T, Deak M, Morrice N, Cohen P. Characterization of the structure and regulation of two novel isoforms of serum- and glucocorticoid-induced protein kinase. Biochem J. 1999;344:189-97.

25. He P, Lee S-J, Lin S, Seidler U, Lang F, Fejes-Toth G, et al. Serum- and glucocorticoid-induced kinase 3 in recycling endosomes mediates acute activation of $\mathrm{Na}+\mathrm{H}+$ exchanger NHE3 by glucocorticoids. Mol Biol Cell. 2011;22:3812-25.

26. Pao AC. SGK regulation of renal sodium transport. Curr Opin Nephrol Hypertens. 2012;21:534-40.

27. Pao AC, McCormick JA, Li H, Siu J, Govaerts C, Bhalla V, et al. $\mathrm{NH} 2$ terminus of serum and glucocorticoid-regulated kinase 1 binds to phosphoinositides and is essential for isoform-specific physiological functions. Am J Physiol Ren Physiol. 2007;292: F1741-50.

28. Pao AC, Bhargava A, Di Sole F, Quigley R, Shao X, Wang J, et al. Expression and role of serum and glucocorticoid-regulated kinase 2 in the regulation of $\mathrm{Na}+/ \mathrm{H}+$ exchanger 3 in the mammalian kidney. Am J Physiol Ren Physiol. 2010;299:F1496-506.

29. Bago R, Sommer E, Castel P, Crafter C, Bailey FP, Shpiro N, et al. The hVps34-SGK3 pathway alleviates sustained PI3K/Akt inhibition by stimulating mTORC1 and tumour growth. EMBO J. 2016;35:2263.

30. Bruhn MA, Pearson RB, Hannan RD, Sheppard KE. AKTindependent PI3-K signaling in cancer - emerging role for SGK3. Cancer Manag Res. 2013;5:281-92. 
31. Castel P, Ellis H, Bago R, Toska E, Razavi P, Carmona FJ, et al. PDK1-SGK1 signaling sustains AKT-independent mTORC1 activation and confers resistance to $\mathrm{PI} 3 \mathrm{~K} \alpha$ inhibition. Cancer Cell. 2016;30:229-42.

32. Vasudevan KM, Barbie DA, Davies MA, Rabinovsky R, McNear CJ, Kim JJ, et al. AKT-independent signaling downstream of oncogenic PIK3CA mutations in human cancer. Cancer Cell. 2009;16:21-32.

33. Sonego M, Pellarin I, Costa A, Vinciguerra GLR, Coan M, Kraut A, et al. USP1 links platinum resistance to cancer cell dissemination by regulating Snail stability. Sci Adv. 2019;5:eaav3235.

34. Pellarin I, Dall'Acqua A, Gambelli A, Pellizzari I, D'Andrea S, Sonego M, et al. Splicing factor proline- and glutamine-rich (SFPQ) protein regulates platinum response in ovarian cancermodulating SRSF2 activity. Oncogene. 2020;39:4390-403.

35. Bruhn MA, Pearson RB, Hannan RD, Sheppard KE. Second AKT: the rise of SGK in cancer signalling. Growth Factors Chur Switz. 2010;28:394-408.

36. Park J, Leong ML, Buse P, Maiyar AC, Firestone GL, Hemmings BA. Serum and glucocorticoid-inducible kinase (SGK) is a target of the PI 3-kinase-stimulated signaling pathway. EMBO J. 1999;18:3024-33.

37. Sherk AB, Frigo DE, Schnackenberg CG, Bray JD, Laping NJ, Trizna W, et al. Development of a small-molecule serum- and glucocorticoid-regulated kinase-1 antagonist and its evaluation as a prostate cancer therapeutic. Cancer Res. 2008;68:7475-83.

38. Sonego M, Pellizzari I, Dall'Acqua A, Pivetta E, Lorenzon I, Benevol S, et al. Common biological phenotypes characterize the acquisition of platinum-resistance in epithelial ovarian cancer cells. Sci Rep. 2017;7:7104.

39. Biazik J, Vihinen H, Anwar T, Jokitalo E, Eskelinen E-L. The versatile electron microscope: an ultrastructural overview of autophagy. Methods San Diego Calif. 2015;75:44-53.

40. Eskelinen E-L. Maturation of autophagic vacuoles in Mammalian cells. Autophagy. 2005;1:1-10.

41. Galluzzi L, Baehrecke EH, Ballabio A, Boya P, Bravo-San Pedro JM, Cecconi F, et al. Molecular definitions of autophagy and related processes. EMBO J. 2017;36:1811-36.

42. Klionsky DJ, Abdelmohsen K, Abe A, Abedin MJ, Abeliovich $\mathrm{H}$, Acevedo Arozena A, et al. Guidelines for the use and interpretation of assays for monitoring autophagy (3rd edition). Autophagy. 2016;12:1-222.

43. Loos B, du Toit A, Hofmeyr J-HS. Defining and measuring autophagosome flux-concept and reality. Autophagy. 2014;10: 2087-96.

44. Mizushima N, Yoshimori T. How to interpret LC3 immunoblotting. Autophagy. 2007;3:542-5.

45. Mizushima N, Yoshimori T, Levine B. Methods in mammalian autophagy research. Cell. 2010;140:313-26.

46. Kimura S, Noda T, Yoshimori T. Dissection of the autophagosome maturation process by a novel reporter protein, tandem fluorescent-tagged LC3. Autophagy. 2007;3:452-60.

47. Katayama H, Yamamoto A, Mizushima N, Yoshimori T, Miyawaki A. GFP-like proteins stably accumulate in lysosomes. Cell Struct Funct. 2008;33:1-12.

48. Thomé MP, Filippi-Chiela EC, Villodre ES, Migliavaca CB, Onzi GR, Felipe KB, et al. Ratiometric analysis of Acridine Orange staining in the study of acidic organelles and autophagy. J Cell Sci. 2016;129:4622-32.

49. Mindell JA. Lysosomal acidification mechanisms. Annu Rev Physiol. 2012;74:69-86.

50. Henke G, Setiawan I, Böhmer C, Lang F. Activation of $\mathrm{Na}+/ \mathrm{K}+$-ATPase by the serum and glucocorticoid-dependent kinase isoforms. Kidney Blood Press Res. 2002;25:370-4.
51. O’Keeffe BA, Cilia S, Maiyar AC, Vaysberg M, Firestone GL. The serum- and glucocorticoid-induced protein kinase-1 (Sgk-1) mitochondria connection: identification of the IF-1 inhibitor of the $\mathrm{F}(1) \mathrm{F}(0)$-ATPase as a mitochondria-specific binding target and the stress-induced mitochondrial localization of endogenous Sgk-1. Biochimie. 2013;95:1258-65.

52. Cotter K, Stransky L, McGuire C, Forgac M. Recent Insights into the structure, regulation, and function of the V-ATPases. Trends Biochem Sci. 2015;40:611-22.

53. Marshansky V, Futai M. The V-type H+-ATPase in vesicular trafficking: targeting, regulation and function. Curr Opin Cell Biol. 2008;20:415-26.

54. Janku F, McConkey DJ, Hong DS, Kurzrock R. Autophagy as a target for anticancer therapy. Nat Rev Clin Oncol. 2011;8:528-39.

55. Levy JMM, Towers CG, Thorburn A. Targeting autophagy in cancer. Nat Rev Cancer. 2017;17:528-42.

56. Rubinsztein DC, Codogno P, Levine B. Autophagy modulation as a potential therapeutic target for diverse diseases. Nat Rev Drug Disco. 2012;11:709-30.

57. Rybstein MD, Bravo-San Pedro JM, Kroemer G, Galluzzi L. The autophagic network and cancer. Nat Cell Biol. 2018;20:243-51.

58. Singh SS, Vats S, Chia AY-Q, Tan TZ, Deng S, Ong MS, et al. Dual role of autophagy in hallmarks of cancer. Oncogene. 2018;37:1142-58

59. Rhodes DR, Yu J, Shanker K, Deshpande N, Varambally R, Ghosh D, et al. ONCOMINE: a cancer microarray database and integrated data-mining platform. Neoplasia N. Y N. 2004;6:1-6.

60. Rhodes DR, Kalyana-Sundaram S, Mahavisno V, Varambally R, $\mathrm{Yu}$ J, Briggs BB, et al. Oncomine 3.0: genes, pathways, and networks in a collection of 18,000 cancer gene expression profiles. Neoplasia N. Y N. 2007;9:166-80.

61. Eskelinen E-L. The dual role of autophagy in cancer. Curr Opin Pharm. 2011;11:294-300.

62. Galluzzi L, Pietrocola F, Bravo-San Pedro JM, Amaravadi RK, Baehrecke EH, Cecconi F, et al. Autophagy in malignant transformation and cancer progression. EMBO J. 2015;34:856-80.

63. Levy JMM, Towers CG, Thorburn A. Targeting autophagy in cancer. Nat Rev Cancer. 2017;17:528-42.

64. Mauvezin C, Nagy P, Juhász G, Neufeld TP. Autophagosomelysosome fusion is independent of V-ATPase-mediated acidification. Nat Commun. 2015;6:7007.

65. Kulshrestha A, Katara GK, Ginter J, Pamarthy S, Ibrahim SA, Jaiswal MK, et al. Selective inhibition of tumor cell associated Vacuolar-ATPase 'a2' isoform overcomes cisplatin resistance in ovarian cancer cells. Mol Oncol. 2016;10:789-805.

66. Song T, Jeon H-K, Hong JE, Choi J-J, Kim T-J, Choi CH, et al. Proton pump inhibition enhances the cytotoxicity of paclitaxel in cervical cancer. Cancer Res Treat J Korean Cancer Assoc. 2017;49:595-606.

67. Lu Z-N, Tian B, Guo X-L. Repositioning of proton pump inhibitors in cancer therapy. Cancer Chemother Pharm. 2017;80: 925-37.

68. Sonego M, Schiappacassi M, Lovisa S, Dall'Acqua A, Bagnoli M, Lovat F, et al. Stathmin regulates mutant p53 stability and transcriptional activity in ovarian cancer. EMBO Mol Med. 2013;5:707-22.

69. Dall'Acqua A, Sonego M, Pellizzari I, Pellarin I, Canzonieri V, D'Andrea S, et al. CDK6 protects epithelial ovarian cancer from platinum-induced death via FOXO3 regulation. EMBO Mol Med. 2017;9:1415-33.

70. Berton S, Pellizzari I, Fabris L, D'Andrea S, Segatto I, Canzonieri $\mathrm{V}$, et al. Genetic characterization of p27(kip1) and stathmin in controlling cell proliferation in vivo. Cell Cycle Georget Tex. 2014;13:3100-11. 